\title{
The Shapley Values on Fuzzy Coalition Games with Concave Integral Form
}

\author{
Jinhui Pang, ${ }^{1}$ Xiang Chen, ${ }^{2}$ and Shujin $\mathrm{Li}^{3}$ \\ ${ }^{1}$ Library, Beijing Institute of Technology, Beijing 100081, China \\ ${ }^{2}$ Patent Examination Cooperation Center of the Patent Office, State Intellectual Property Office of P.R.C, Beijing 100088, China \\ ${ }^{3}$ Department of Information Management, The Central Institute for Correctional Police, Baoding 071000, China
}

Correspondence should be addressed to Jinhui Pang; pangjinhui69@126.com

Received 15 November 2013; Accepted 15 December 2013; Published 27 January 2014

Academic Editor: Pu-yan Nie

Copyright (C) 2014 Jinhui Pang et al. This is an open access article distributed under the Creative Commons Attribution License, which permits unrestricted use, distribution, and reproduction in any medium, provided the original work is properly cited.

\begin{abstract}
A generalized form of a cooperative game with fuzzy coalition variables is proposed. The character function of the new game is described by the Concave integral, which allows players to assign their preferred expected values only to some coalitions. It is shown that the new game will degenerate into the Tsurumi fuzzy game when it is convex. The Shapley values of the proposed game have been investigated in detail and their simple calculation formula is given by a linear aggregation of the Shapley values on subdecompositions crisp coalitions.
\end{abstract}

\section{Introduction}

There are many solution concepts in crisp cooperative game which are allocations for the profits of a cooperative game, such as Shapley value [1], Banzhaf value [2], and $\tau$-value [3]. However, there are many possible or vague factors that might influence players' decisions such that the cooperation is full of uncertainty. As a result, these solutions are not suitable to games with vague factors.

In fuzzy environment, fuzzy cooperative games theory extends crisp games' results by using fuzzy set theory (such as Zadeh [4], Mareš [5], and Dubois and Prade [6]). It also focuses on the problems of how to express fuzzy coalitions, how to evaluate fuzzy payoffs, and how to distribute it among players.

Nowadays the fuzzy games mainly consist of two types. One is games with fuzzy coalitions, in which players partly take part in a coalition, but exact profits of fuzzy coalitions can be gained. For examples, Aubin [7] and Butnariu [8] defined a fuzzy game whose character function was the aggregated worth of the coalitions profits with respect to players' participation degree. The other is games with fuzzy payoffs, a game with fuzzy payoffs but the coalitions are still crisp game coalitions. For examples, Mareš $[9,10]$ and Mareš and Vlach [11] suggested that the values assigned to coalitions were fuzzy quantities even though the domain of the character function of fuzzy games remained to be accurate like crisp games.

In games with fuzzy coalitions literature, Tsurumi et al. [12] pointed out shortcomings of the game proposed by Aubin and Butnariu and proposed a class of fuzzy games by the Choquet integral. Borkotokey [13] took a cooperative game with fuzzy coalitions and fuzzy character functions into consideration simultaneously, where character functions were fuzzy value which mapped the set of real numbers to the closed interval $[0,1]$.

At present, the Shapley values of fuzzy games have been studied by many scholars after Butnariu [14] who firstly defined fuzzy Shapley function on a limited class of fuzzy games with proportional values. But it was neither monotone nondecreasing nor continuous with regard to rates of players' participation. Later, Butnariu and Kroupa [15] similarly gave the Shapley values on fuzzy games with weighted function. Tsurumi et al. also discussed the Shapley values on their fuzzy game, which was both monotone nondecreasing and continuous with regard to players' participation rates because of the advantageous properties of the Choquet integral. Borkotokey [13] discussed its Shapley value on games whose payoffs and coalitions are both fuzzy. 
Actually, players mostly prefer to estimate cooperative profits on their vague knowledge of the game. Based on this, integrals with fuzzy measures or fuzzy capacities should be suitable to model fuzzy games. The integral theory had made use of a cooperative game that appeared in Weber [16] and Azrieli and Lehrer [17]. Besides Tsurumi et al. [12], Dow and Werlang $[18,19]$ applied the Choquet integral to game theory and finance. In many cases, fuzzy capacities assign decisionsubjective expected values to some coalitions but not to all. Therefore, we will at first introduce a new cooperative game form by the Concave integral with respect to fuzzy capacity, which has been discussed in detail by Lehrer [20], and will investigate the Shapley values on the new fuzzy cooperative games in detail.

The lecture will be organized as follows. In Section 2, we will recall the concepts of crisp cooperative game and its Shapley values will also be recalled. In Section 3, some games with fuzzy coalitions and several game forms, such as Butnariu and Tsurumi fuzzy game, will be given. Moreover, we define a new fuzzy game by the Concave integral and its several properties will also be provided. Meanwhile, we illustrate that the proposed game is an extension of Tsurumi fuzzy game. In Section 4, we discuss the Shapley values for the new game, which can be gained by a linear aggregation of the Shapley values on subdecompositions crisp coalitions. Finally, some conclusions appear in Section 5.

\section{Crisp Cooperative Game and the Shapley Value}

A finite set of players $N=\{1,2, \ldots, n\}$ is a nonempty set, in which players may take part in different feasible subcoalition of $N$. The greatest coalition $N$ is the grand set and the smallest coalition is $\phi$. The power set $P(S)$ is the family of all crisp subcoalitions of $S \subseteq N$.

A crisp cooperative game on player set $N$ is denoted by $v$ where the character function $v: P(N) \rightarrow R_{+} \cup\{0\}$ with $v(\phi)=0$. For any a $S \in P(N)$, the worth $v(S)$ assigning to $S$ can be regarded as the maximal worth or cost of the coalition $S$ which is obtained when players in $S$ work together. We take the notation $G(N, v)$ to express the class of all crisp games with player set $N$.

For a nonempty subset $S \in P(N)$, the simple games $u_{S}$ are defined by

$$
u_{S}(A)= \begin{cases}1, & \text { if } S \subseteq A, \\ 0, & \text { otherwise }\end{cases}
$$

And each cooperative game $v \in G(N, v)$ can be represented by $u_{S}$ as follows:

$$
v=\sum_{T \in P(N) \backslash\{\phi\}} c_{S}(v) u_{S},
$$

where $c_{S}(v)=\sum_{B \in P(N): B \subseteq S}(-1)^{|S|-|B|} v(B)$.

The game $v \in G(N, v)$ is said to be convex when

$$
v(S \cup T)+v(S \cap T) \geq v(S)+v(T), \quad \forall S, T \in P(N) .
$$

The convex game $v \in G(N, v)$ is said to be superadditive, if any disjoint crisp coalitions $S$ and $T$ satisfy

$$
v(S \cup T) \geq v(S)+v(T), \quad \forall S, T \in P(N), S \cap T=\phi .
$$

The notation $G_{0}(N, v)$ represents all superadditave crisp cooperative games. For the game $v \in G(N, v)$, players $i$ and $j$ are said to be symmetric, if $S \subseteq N \backslash\{i, j\}$ such that

$$
v(S \cup i)=v(S \cup j) .
$$

The player $i \in N$ is a dummy player of the game, if for any $S \subseteq N \backslash\{i\}$ such that

$$
v(S \cup i)=v(S)+v(i) .
$$

The player $i \in N$ is a null player in a coalition $W$ for a game $v \in G_{0}(N, v)$, if

$$
v(S)=v(S \cup\{i\}), \quad \forall S \in P(W \backslash\{i\}) .
$$

Definition 1. An imputation for a crisp cooperative game $v \in$ $G(N, v)$ is a vector $x=\left(x_{1}, x_{2}, \ldots, x_{n}\right) \in R_{+}^{n} \cup\{0\}$ satisfying

(1) $\sum_{i \in N} x_{i}=v(N)$,

(2) $x_{i} \geq v(i), \forall i \in N$.

It is obvious that $v \in G_{0}(N, v)$ when imputation for a crisp cooperative game $v \in G(N, v)$ is nonempty.

Definition 2. Let $G_{0}(N, v), W \in P(N)$; then $S \in P(W)$ is called a carrier in a coalition $W$ for a game $v$ if

$$
v(S \cap T)=v(T), \quad \forall T \in P(W) .
$$

If the set of all carriers in coalition $W$ for $v$ is denoted by $C(W \mid v)$, then

$$
C(W \mid v)=\{S \in P(N) \mid v(S \cap T)=v(T), \forall T \in P(N)\} .
$$

A well-known solution for cooperative game $v \in$ $G_{0}(N, v)$, the Shapley value is a mathematical expectation on $P(N \backslash\{i\})$ with regard to marginal contribution $v(T \cup i)-v(T)$ where $T \subseteq N \backslash\{i\}$. Shapley [1] defined the function satisfying the following 4 axioms.

Definition 3. A function $f^{\prime}: G_{0}(n, v) \rightarrow\left(R_{+}^{n}\right)^{P(N)}$ is said to be a Shapley value on $G_{0}(N, v)$ if it satisfies the following four axioms.

Axiom 1. If $v \in G_{0}(N, v)$ and $W \in P(N)$, then

$$
\begin{gathered}
\sum_{i \in N} f_{i}^{\prime}(v)(W)=v(W), \\
f_{i}^{\prime}(v)(W)=0, \quad \forall i \notin W,
\end{gathered}
$$

where $f_{i}^{\prime}(v)(W)$ is the $i$ th element of $f^{\prime}(v)(W) \in R_{+}^{n}$.

Axiom 2. If $v \in G_{0}(N, v), W \in P(N)$, and $T \in C(W \mid v)$, then

$$
f_{i}^{\prime}(v)(W)=f_{i}^{\prime}(v)(T), \quad \forall i \in N .
$$


Axiom 3. If $v \in G_{0}(N, v), W \in P(N), i, j \in W$, and $v(S \cup\{i\})=$ $v(S \cup\{j\})$ for any $S \in P(W \backslash\{i, j\})$, then

$$
f_{i}^{\prime}(v)(W)=f_{j}^{\prime}(v)(W) .
$$

Axiom 4. For any $v_{1}, v_{2} \in G_{0}(N, v)$, define a game $v_{1}+v_{2} \in$ $G_{0}(N, v)$ by $\left(v_{1}+v_{2}\right)(S)=v_{1}(S)+v_{2}(S)$ for any $S \in P(N)$. If $v_{1}, v_{2} \in G_{0}(N, v)$ and $W \in P(N)$, then

$$
f_{i}^{\prime}\left(v_{1}+v_{2}\right)(W)=f_{i}^{\prime}\left(v_{1}\right)(W)+f_{i}^{\prime}\left(v_{2}\right)(W), \quad \forall i \in N .
$$

Shapley [1] also gave the uniquely explicit form of a Shapley value on $G_{0}(N, v)$ which was obtained by extending the Shapley value for the grand coalition $N$ as below.

Theorem 4. Define a function $f^{\prime}: G_{0}(N, v) \rightarrow\left(R_{+}^{n}\right)^{P(N)}$ by

$$
f_{i}^{\prime}(v)(W)= \begin{cases}\sum_{T \in P_{i}(W)} \beta(|T| ;|W|) & \\ \cdot\{v(T)-v(T \backslash\{i\})\}, & \text { if } i \in W, \\ 0, & \text { otherwise, }\end{cases}
$$

where $P_{i}(W)=\{i \in T \mid T \in P(W)\}, \beta(|T| ;|W|)=(|T|-1) ! \cdot$ $(|W|-|T|) ! /|W| !$ and $|\cdot|$ is the number of players in set $\cdot$. Then the function $f^{\prime}$ is the unique Shapley function on $G_{0}(N, v)$.

It is obvious that $f^{\prime}(v)$ is an imputation of the cooperative game $G_{0}(N, v)$. Meanwhile, if $v \in G_{0}(N, v)$ is convex, then $i \in$ $N$ and $S \subseteq T$ imply that $f_{i}^{\prime}(v)(S) \leq f_{i}^{\prime}(v)(T)$.

\section{Fuzzy Coalition Games}

Let us start by presenting some general definitions related to fuzzy coalition games.

3.1. Basic Concepts. We consider cooperative fuzzy games with the player set $N=\{1,2, \ldots, n\}$. A fuzzy coalition $\widetilde{S}$ is a fuzzy subset of the finite set $N$, which is a vector $s=$ $\left(s_{1}, s_{2}, \ldots, s_{n}\right)$ where $s_{i} \in[0,1]$ describes the membership grade of player $i$ in the fuzzy coalition $\widetilde{S}$. We note that a different coalition has different vector $s=\left(s_{1}, s_{2}, \ldots, s_{n}\right)$, so we also call it as fuzzy coalition variable. If element $s_{i}=1$ when $i$ fully take part in $\widetilde{S}$ and others $s_{i}=0$, then the coalition $\widetilde{S}$ is a crisp coalition.

Consider the crisp coalition $e^{k}=\{0,0, \ldots, 1, \ldots, 0\}$ where the $k$ th element is 1 and others are zero. The fuzzy coalition variable $s=\left(s_{1}, s_{2}, \ldots, s_{n}\right)$ has the regular form

$$
s=\sum_{i \in N} s_{i} e^{i}
$$

For the fuzzy coalition $\widetilde{S}$ and $\widetilde{U}$ with fuzzy coalition variables vector $s$ and $u, s_{i} \leq u_{i}(\forall i \in N)$ if and only if $\widetilde{S} \subseteq \widetilde{U}$. The class of all fuzzy coalitions in $\widetilde{U}$ is denoted by $L(\widetilde{U})$; that is, $L(\widetilde{U})=\{\widetilde{S} \mid \widetilde{S} \subseteq \widetilde{U}\}$. The level set of fuzzy coalition $\widetilde{S}$ is the set $[\widetilde{S}]_{t}=\left\{i \in N \mid s_{i} \geq t\right\}$; its $t$-section is the set $\widetilde{S}_{t}=\left\{i \mid i \in N, s_{i}=t\right\}$ which is a player set with the same level $r$, and its support set is the $\operatorname{set} \operatorname{Supp}(\widetilde{S})=\left\{i \in N \mid s_{i}>0\right\}$.
A fuzzy coalition game $\widetilde{v}$ is the function $\widetilde{v}: L(N) \rightarrow R_{+} U$ $\{0\}$ with $v(\phi)=0$. We take the notation $G(N, \widetilde{v})$ as the class of all fuzzy coalition games $\widetilde{v}$. We call $v \in G(N, \widetilde{v})$ continuous, for any two fuzzy coalitions $\widetilde{S}$ and $\widetilde{T}$ with variables $s=$ $\left(s_{1}, s_{2}, \ldots, s_{n}\right)$ and $t=\left(t_{1}, t_{2}, \ldots, t_{n}\right)$, respectively, if

$$
\lim _{d \rightarrow 0} v\left(\sum_{i \in S} s_{i} e^{i}\right)=v\left(\sum_{i \in T} s_{i} e^{i}\right),
$$

where $d=\max _{i \in N}\left|s_{i}-t_{i}\right|$. A game $v \in G(N, \widetilde{v})$ is said to be monotonic, for every fuzzy coalition $\widetilde{S}$ and $\widetilde{U}, \widetilde{S} \subseteq \widetilde{U}$ implies $v(\widetilde{S}) \leq v(\widetilde{U})$.

In this paper, we assume that every fuzzy coalition variables maps into the lattice $([0,1], \wedge, \vee)$, where $\wedge$ and $\vee$ are the minimum and maximum operators, respectively. For any fuzzy coalition $\widetilde{S}, \widetilde{U} \in L(N)$, we adopt the usual definition of the union and intersection of fuzzy subsets given by

$$
\begin{aligned}
& (\widetilde{S} \cup \widetilde{U})(i)= \begin{cases}s_{i} \vee u_{i}, & i \in \operatorname{Supp} \widetilde{S} \cup \operatorname{Supp} \widetilde{U}, \\
0, & \text { others, }\end{cases} \\
& (\widetilde{S} \cap \widetilde{U})(i)= \begin{cases}s_{i} \wedge u_{i}, & i \in \operatorname{Supp} \widetilde{S} \cap \operatorname{Supp} \widetilde{U} \\
0, & \text { others. }\end{cases}
\end{aligned}
$$

Similarly to crisp convex game, for all $\widetilde{S}, \widetilde{U} \in L(N), v \in$ $G(N, \widetilde{v})$ is said to be fuzzy convex, if it satisfies

$$
v(\widetilde{S})+v(\widetilde{U}) \leq v(\widetilde{S} \cup \widetilde{U})+v(\widetilde{S} \cap \widetilde{U}),
$$

and $\widetilde{v} \in G(N, \widetilde{v})$ is said to be superadditive such that

$$
v(\widetilde{S} \cup \widetilde{U}) \geq v(\widetilde{S})+v(\widetilde{U})
$$

with $\widetilde{S} \cap \widetilde{U}=\phi$.

Definition 5. A function $y: L(\widetilde{U}) \rightarrow R_{+}^{n}$ is said to be an imputation for a fuzzy game $v \in G(N, \widetilde{v})$ in fuzzy coalition $\widetilde{U} \in L(N)$ with fuzzy coalition variable $u=\left(u_{1}, u_{2}, \ldots, u_{n}\right)$, if

(1) $y_{i}(\widetilde{U})=0, \forall i \notin \operatorname{Supp}(\widetilde{U})$,

(2) $\sum_{i \in N} y_{i}(\widetilde{U})=v(\widetilde{U})$,

(3) $y_{i}(\widetilde{U}) \geq u_{i} v\left(e^{i}\right)$,

where $y_{i}(\widetilde{U})=\left(y_{1}(\widetilde{U}), y_{2}(\widetilde{U}), \ldots, y_{n}(\widetilde{U})\right)$.

Definition 6. Let $v \in G(N, \widetilde{v})$; the player $i \in N$ is said to be a dummy player on fuzzy coalition $\widetilde{U} \in L(N)$ with fuzzy coalition variable $u=\left(u_{1}, u_{2}, \ldots, u_{n}\right)$, if for any fuzzy coalition $\widetilde{S}$,

$$
v\left(\left(\sum_{j \in \operatorname{Supp} S \backslash i} s_{j} e^{j}\right) \cup s_{i} e^{i}\right)=v\left(\sum_{j \in \operatorname{Supp} S \backslash i} s_{j} e^{j}\right)+v\left(s_{i} e^{i}\right)
$$

and if

$$
v\left(\left(\sum_{j \in \operatorname{Supp} S \backslash i} s_{j} e^{j}\right) \cup s_{i} e^{i}\right)=v\left(\sum_{j \in \operatorname{Supp} S \backslash i} s_{j} e^{j}\right) .
$$

The player $i$ is called a null player on fuzzy coalition $\widetilde{U}$. 
Example 7. Let $N=\{1,2,3\}$ and for any fuzzy coalition variable $x=\left(x_{1}, x_{2}, x_{3}\right)$, define $v(x) \in G(N, \widetilde{v})$ by

$$
v(x)= \begin{cases}x_{1}+x_{2}+x_{3}, & \text { if } x_{3} \geq 0.4 \\ x_{1}+x_{2}, & \text { otherwise }\end{cases}
$$

Suppose a fuzzy coalition $\widetilde{U} \in L(N)$ with the fuzzy variable value $u=(0.4,0.6,0.7)$; then for any fuzzy coalition $\widetilde{S}$ with fuzzy variable value $s=\left(s_{1}, s_{2}, s_{3}\right)$, player 3 is a dummy player when $s_{3}<0.4$ and he is a null player when $s_{3} \geq 0.7$.

Definition 8. Let $v \in G(N, \widetilde{v}), \widetilde{U} \in L(N)$; then $\widetilde{S} \in L(\widetilde{U})$ is called a fuzzy carrier in a coalition $\widetilde{U}$ if for any $\forall \widetilde{T} \in L(\widetilde{U})$ such that

$$
v(\widetilde{S} \cap \widetilde{T})=v(\widetilde{T}) .
$$

The set of all carriers in fuzzy coalition $\widetilde{U}$ for $v \in G(N, \widetilde{v})$ is denoted by $C(\widetilde{U} \mid v)$; it is obvious that

$$
C(\widetilde{U} \mid v)=\{\widetilde{S} \in L(\widetilde{U}) \mid v(\widetilde{S} \cap \widetilde{T})=v(\widetilde{T}), \forall \widetilde{T} \in L(\widetilde{U})\} .
$$

3.2. The Present Forms for Fuzzy Coalition Games. In the field of fuzzy cooperative games with fuzzy coalitions, there were several definitions given by aggregating function on fuzzy coalition variables, such as Butnariu game, Butnariu and Kroupa game, and Tsurumi game.

In Butnariu game, $v(\widetilde{S})$ was an aggregated worth of the crisp coalitions $\widetilde{S}_{t}$ where the players have the same participation level $t$, defined by

$$
v(\widetilde{S})=\sum_{t \in[0,1]} v\left(\widetilde{S}_{t}\right) \cdot t, \quad \forall \widetilde{S} \in L(N) .
$$

It is obvious that the game value is a linear aggregation function which is a weighted average on the sets with the same participation levels, namely, a fuzzy game with proportional values as the associated crisp game. We denote the fuzzy game with proportional values as the notation $G^{P}(N)$. It is a one-toone correspondence between a crisp game and a fuzzy game with proportional values.

Butnariu and Kroupa [15] proposed a fuzzy game model with weight function as follows:

$$
v(\widetilde{S})=\sum_{t \in[0,1]} \psi(t) v\left(\widetilde{S}_{t}\right)
$$

where $\psi:[0,1] \rightarrow R$ is a function with the properties $\psi(t)=$ $0 \Leftrightarrow t=0$ and $\psi(1)=1$.

Similarly, it is also a simple linear aggregation function which cannot embody the interaction among players with different participation levels. Moreover, if $\psi(t)=t$ implies that the game is equivalent to the proportional game, we denote it as $G^{\psi}(N)$.

Tsurumi et al. introduced another form definition based on the Choquet integral, which was not only monotone nondecreasing but also continuous with regard to rates of players' participation. Let $\widetilde{S} \in L(N), Q(\widetilde{S})=\left\{s_{i} \mid s_{i}>0, i \in\right.$ $N\}$ and rearrange elements in $Q(\widetilde{S})$ such that $0=h_{0} \leq h_{1}<$ $h_{2}<\cdots<h_{q(\widetilde{S})}$; then for any $\widetilde{S} \in L(N)$, a game $v: L(N) \rightarrow R$ is defined by

$$
v(\widetilde{S})=\sum_{l=1}^{q(\widetilde{S})} v\left([\widetilde{S}]_{h_{l}}\right) \cdot\left(h_{l}-h_{l-1}\right),
$$

where $q(\widetilde{S})$ is the cardinality of $Q(\widetilde{S})$.

The fuzzy game given by Tsurumi et al. is simply denoted by $G^{\mathrm{Ch}}(N)$. It is apparent that the fuzzy game $v \in G^{\mathrm{Ch}}(N)$ is a Choquet integral of the function $h$ with respect to $v$ derived from level set. We note that $h_{l-1}<h_{l}$ implies that $[\widetilde{S}]_{h_{l}} \subseteq$ $[\widetilde{S}]_{h_{l-1}}$, so the worth of coalition $\widetilde{S}$ is the maximum sum on all subsets which is an including chain.

Example 9. Let $N=\{1,2,3\}$ and let $v$ be a character function on $N$ which is joint workers' output. $v(1)=v(2)=v(3)=2$, $v(1,3)=8, v(1,2)=9, v(2,3)=5$, and $v(1,2,3)=10$.

Suppose that the fuzzy coalition $s=(1,0.4,0.6)$; rearrange it as $0.4<0.6<1$; thus, the value of this fuzzy coalition is evaluated by (27) as follows:

$$
\begin{aligned}
v(\widetilde{S})= & \sum_{l=1}^{q(\widetilde{S})} v\left([\widetilde{S}]_{h_{l}}\right) \cdot\left(h_{l}-h_{l-1}\right) \\
= & v\left([\widetilde{S}]_{0.4}\right) \times 0.4+v\left([\widetilde{S}]_{0.6}\right) \\
& \times(0.6-0.4)+v\left([\widetilde{S}]_{1}\right) \times(1-0.6) \\
= & v(1,2,3) \times 0.4+v(1,3) \\
& \times 0.2+v(1) \times 0.4=6.4 .
\end{aligned}
$$

However, there are another linear aggregation values which are greater than that of Tsurumi's form. For example, we make a linear sum as

$$
v(\widetilde{S})=0.4 \times v(1,2)+0.6 \times v(1,3)=8.4 .
$$

Hence, Tsurumi's class cannot be considered as an optimal product on $P(N)$, for fuzzy variables assign subjective expected values to some coalitions but not to all in Tsurumi game. As a result Tsurumi fuzzy game is not suitable in some situations.

3.3. A Class of Fuzzy Coalition Games with the Concave Integral. As mentioned above, the present forms for fuzzy coalition games were only limited to some special games and will be invalid in many game situations. Next, we will consider another extended game with fuzzy coalitions, that is, the fuzzy game with the Concave integral, where Tsurumi game can be taken as a special case as the proposed new game. Firstly, we recall the fuzzy capacity and the Concave integral.

Let $N$ be a finite set $(|N|=n)$; a capacity $\mu$ over $N$ is a function $\mu: P(N) \rightarrow R^{+} \cup\{0\}$ such that $S \subseteq T \subseteq N$ implies $\mu(S) \leq \mu(T)$ with $\mu(\phi)=0$. A random variable over $N$ is 
a function $X: N \rightarrow R$ and a random variable is nonnegative if $x_{i} \geq 0$ for every $i \in N$.

We proposed fuzzy capacity game concept defined by the following way.

Definition 10. Let $\widetilde{A} \in L(N)$; the pair $(v, \widetilde{A})$ is said to be a fuzzy capacity game if $v: L(N) \rightarrow R^{+} \cup\{0\}$ is monotonic and continuous, and there is a positive $M$ such that $v(a) \leq M|a|$ for every $a \in L(\widetilde{A})$.

Definition 11. Let $\widetilde{A} \in L(N)$; the pair $(v, \widetilde{A})$ is said to be an additive fuzzy capacity game, for every fuzzy coalition variable $a \in L(\widetilde{A})$, if there is a nonnegative constant vector $p=$ $\left(p_{1}, p_{2}, \ldots, p_{n}\right)$ such that $v(a)=\sum_{i=1}^{n} a_{i} p_{i}$.

It is not hard to see that the limited game given by Butnariu is an additive fuzzy capacity game. A fuzzy capacity game assigns values (subjective expected value) to fuzzy coalition random variables, in which players express their preferences of some coalitions but not of all. The fuzzy coalition $\widetilde{A}$ might contain only extreme or discrete points of the domain of $L(\widetilde{S})$ where $\widetilde{S} \subseteq L(N)$ such as $(1,1, \ldots, 1)$ and $(0,0, \ldots, 0)$, therefore $v$ may be partially nonadditive or nonadditive on its domains.

The integral aggregates all available fuzzy coalitions, including individual assessments of the likelihood of events and expected values of variables, into a comprehensive value. By this value, the players reevaluate their likely coalitions or expected values on random coalition variables.

Let $s$ be a random variable; a subdecomposition of $s$ is a finite summation $\sum_{A_{i} \subseteq N} \alpha_{i} A_{i}$ that satisfies

$$
\begin{gathered}
\sum_{A_{i} \subseteq N} \alpha_{i} 1_{A_{i}} \leq s, \quad\left(\alpha_{i} \geq 0\right), \\
A_{i} \subseteq N, \quad\left(i=1,2,3, \ldots, 2^{n}\right) .
\end{gathered}
$$

Definition 12 . Let $v \in G(N, \widetilde{v})$ be a fuzzy capacity game, let $\widetilde{S} \in$ $L(N)$ be a random fuzzy coalition with nonnegative variable $s=\left(s_{1}, s_{2}, \ldots, s_{n}\right)$, and define a game $v^{\mathrm{Cav}}: L(N) \rightarrow R_{+} \cup\{0\}$ by

$$
v^{\mathrm{Cav}}(\widetilde{S})=\int^{\mathrm{Cav}} s d v=\min \{f(s)\}
$$

where the minimum is taken all over concave and homogeneous functions $f: R_{+}^{n} \rightarrow R$ and $f\left(1_{T}\right) \geq v(T)$ for every $T \subseteq$ $N$, and $1_{T}$ is the indicator of $T$ which is the random variable that takes the value 1 over $T$ and the value 0 , otherwise.

By Definition 12, $v^{\mathrm{Cav}}(\widetilde{S})$ can be gained by the values on crisp coalitions which correspond with subdecompositions of $s$.

We denote all fuzzy games defined by the concave integral as $G^{\mathrm{Cav}}(N)$.

From the above definition, the function $f$ is defined on all over concave coalitions. It is easy to prove the following lemma.
Lemma 13. Let $v \in G(N, \widetilde{v})$ be a fuzzy capacity game; for every random fuzzy coalition $\widetilde{S} \in L(N)$ with nonnegative variable $s=\left(s_{1}, s_{2}, \ldots, s_{n}\right)$,

$$
\int^{C a v} s d v=\max \left\{\sum_{T \subseteq N} \alpha_{T} v(T), \sum_{T \subseteq N} \alpha_{T} 1_{T}=s, \alpha_{T} \geq 0\right\} .
$$

The game $v^{\text {Cav }}: L(N) \rightarrow R_{+} \cup\{0\}$ can also be calculated by

$$
\begin{array}{ll}
v^{\operatorname{Cav}}(\widetilde{S})=\int^{C a v} s d v=\max \sum_{T_{i} \subseteq N} \alpha_{T_{i}} v\left(T_{i}\right) \\
\text { s.t. } \quad \sum_{T_{i} \subseteq N} \alpha_{T_{i}} 1_{T_{i}}=s, \\
& T_{i} \subseteq N, \quad\left(i=1,2,3, \ldots, 2^{n}\right), \\
& \alpha_{T_{i}} \geq 0, \quad\left(i=1,2,3, \ldots, 2^{n}\right) .
\end{array}
$$

Remark 14. When $S \subseteq N, \int^{\mathrm{Cav}} 1_{S} d v=\max \left\{\sum_{T \subseteq N} v(T)\right.$, $\left.\sum_{T \subseteq N} 1_{T}=1\right\}=v(S)$. It is apparent that the fuzzy game with the concave integral extends the crisp game.

Example 15. Consider again Example 9; for the fuzzy coalition $s=(1,0.4,0.6)$, by inequality (33), we have

$$
\begin{array}{ll}
v^{\mathrm{Cav}} & (\widetilde{S})=\int s d v=\max \sum_{T_{i} \subseteq N} \alpha_{T_{i}} v\left(T_{i}\right) \\
\text { s.t. } & \sum_{T_{i}} \alpha_{T_{i}} 1_{T_{i}}=(1,0.4,0.6), \\
& T_{i} \in\{\{1\},\{2\},\{3\},\{1,3\},\{1,2\},\{2,3\},\{1,2,3\}\}, \\
& \alpha_{T_{i}} \in\{0.4,0.6,1\} .
\end{array}
$$

Hence,

$$
T_{1}=\{1,2\}, \quad T_{2}=\{1,3\}, \quad \alpha_{T_{1}}=0.4, \quad \alpha_{T_{2}}=0.6 .
$$

So

$$
v^{\mathrm{Cav}}(\widetilde{S})=0.4 \times 9+0.6 \times 8=8.4 .
$$

We know that $v^{\mathrm{Cav}}(\widetilde{S})=\int{ }^{\mathrm{Cav}} s d v$ is the maximum of the values $\sum_{i=1}^{k} \alpha_{T_{i}} \mu\left(T_{i}\right)$ among all possible decompositions of $\widetilde{S}$ with the coalition variable $s$. The maximum focuses all possible decompositions rather than restrict viable decompositions like the fuzzy game given by the Choquet integral.

In the fuzzy game given by Tsurumi et al., the Choquet integral of nonnegative $X$ with respect to a capacity $v$ is defined by

$$
\int^{\mathrm{Ch}} X d \mu=\sum_{i=1}^{n}\left(X_{\sigma(i)}-X_{\sigma(i-1)}\right) v\left(Q_{i}\right),
$$


where $\sigma$ is a permutation on $N$ such that $0=X_{\sigma(0)} \leq X_{\sigma(1)} \leq$ $X_{\sigma(2)} \leq \cdots \leq X_{\sigma(n)}$ and $Q_{i}=\{\sigma(i), \sigma(2), \ldots, \sigma(n)\}$.

Let $\alpha_{i}=X_{\sigma(i)}-X_{\sigma(i-1)}$; note that $X=\sum \alpha_{i} 1_{Q_{i}}$ is a decomposition of $X$. That is to say that the Choquet integral is defined under the special decomposition of $X$. By contrast, all possible decompositions are allowed in the concave integral. By this way, it implies that $\int^{\text {Ch }} X d \mu \leq \int^{\text {Cav }} X d \mu$ for any $X$. In addition, it has been proven that $\int^{\mathrm{Ch}} X d \mu=\int^{\mathrm{Cav}} X d \mu$ if and only if $\mu$ is convex (see [17]).

Lemma 16. Let $v \in G^{\text {Cav }}(N)$ be a fuzzy capacity game, and let $\widetilde{S} \in L(N)$ be a random fuzzy coalition with nonnegative variable $s=\left(s_{1}, s_{2}, \ldots, s_{n}\right)$; then

$$
v^{C h}(\widetilde{S})=\int^{C h} s d v=\max \sum_{T_{i} \subseteq N} \alpha_{T_{i}} v\left(T_{i}\right),
$$

where $T_{j} \subseteq T_{i}(\forall i<j)$.

Theorem 17. Let a fuzzy capacity game $v \in G^{\text {Cav }}(N)$; then $v$ is continuous with respect to the fuzzy coalition variables.

Proof. For any two nonnegative random fuzzy coalitions $\widetilde{U}$ and $\widetilde{S}$ with variables $u$ and $s$, define a distance $d$ on $L(N)$ by

$$
d(\widetilde{S}, \widetilde{U})=\max _{i \in N}\left|s_{i}-u_{i}\right|
$$

We have

$$
\begin{aligned}
d\left(v^{\text {Cav }}(\widetilde{S}), v^{\text {Cav }}(\widetilde{U})\right) \\
=\left|v^{\text {Cav }}(\widetilde{S})-v^{\text {Cav }}(\widetilde{U})\right| \\
=\left|\max \sum_{S_{i} \subseteq N} \alpha_{S_{i}} v\left(S_{i}\right)-\max \sum_{U_{i} \subseteq N} \alpha_{U_{i}}^{\prime} v\left(U_{i}\right)\right| \\
\leq \max \left|\sum_{S_{i} \subseteq N} \alpha_{S_{i}} v\left(S_{i}\right)-\sum_{U_{i} \subseteq N} \alpha_{U_{i}}^{\prime} v\left(U_{i}\right)\right| \\
\leq \max _{S_{i} \subseteq N, U_{i} \subseteq N} \sum\left|\alpha_{S_{i}} v\left(S_{i}\right)-\alpha_{U_{i}}^{\prime} v\left(U_{i}\right)\right| \\
\leq \sum_{S_{i} \subseteq N, U_{i} \subseteq N}\left(v\left(S_{i}\right), v\left(U_{i}\right)\right)\left|\alpha_{S_{i}}-\alpha_{U_{i}}^{\prime}\right| \\
\leq \sum_{S_{i} \subseteq N, U_{i} \subseteq N}\left(v\left(S_{i}\right), v\left(U_{i}\right)\right) \max _{i \in N}\left|s_{i}-u_{i}\right| \\
\leq d(\widetilde{S}, \widetilde{T}) \sum_{S_{i} \subseteq N, U_{i} \subseteq N}\left(v\left(S_{i}\right), v\left(U_{i}\right)\right) .
\end{aligned}
$$

Since there exists a constant $M$ such that $\sum \max _{S_{i} \subseteq N, U_{i} \subseteq N}$ $\left(v\left(S_{i}\right), v\left(U_{i}\right)\right) \leq M$, we get

$$
d\left(v^{\operatorname{Cav}}(\widetilde{S}), v^{\operatorname{Cav}}(\widetilde{U})\right) \leq M d(\widetilde{S}, \widetilde{U}) .
$$

Therefore, when $d(\widetilde{S}, \widetilde{U}) \rightarrow 0$ then $d\left(v^{\text {Cav }}(\widetilde{S}), v^{\text {Cav }}(\widetilde{U})\right) \rightarrow$ 0 .
Example 18. Continuing with Example 9, for another fuzzy coalition $\widetilde{U}$ with $u=(0.4,1,0.6)$, by inequality (33), we have

$$
\begin{aligned}
v^{\mathrm{Cav}}(\widetilde{U}) & =\int^{\mathrm{Cav}} u d v=\max \sum_{T_{i} \subseteq N} \alpha_{T_{i}} v\left(T_{i}\right) \\
& =0.4 v(1,2)+0.6 v(2,3)=6.6 .
\end{aligned}
$$

By (27), we also get

$$
\begin{aligned}
v^{\mathrm{Ch}}(\widetilde{U})= & \int^{\mathrm{Ch}} u d v=\sum_{l=1}^{q(\widetilde{U})} v\left([\widetilde{U}]_{h_{l}}\right) \cdot\left(h_{l}-h_{l-1}\right) \\
= & v\left([\widetilde{U}]_{0.4}\right) \times 0.4+v\left([\widetilde{U}]_{0.6}\right) \\
& \times(0.6-0.4)+v\left([\widetilde{U}]_{1}\right) \times(1-0.6) \\
= & 0.4 v(1,2,3)+0.2 v(2,3)+0.4 v(2)=5.8 .
\end{aligned}
$$

From the above examples, we note that $v^{\mathrm{Cav}}(\widetilde{S}) \geq v^{\mathrm{Cav}}(\widetilde{U})$ while $v^{\mathrm{Ch}}(\widetilde{S}) \geq v^{\mathrm{Ch}}(\widetilde{U})$.

Theorem 19. Let $v \in G(N, \widetilde{v})$ be a fuzzy capacity game; $v$ is convex if and only if for any two nonnegative random fuzzy coalitions $\widetilde{U}$ and $\widetilde{S}$ with variables $u$ and s, respectively, $v^{\text {Cav }}(\widetilde{S}) \geq$ $v^{C a v}(\widetilde{U})$ whenever $v^{C h}(\widetilde{S}) \geq v^{C h}(\widetilde{U})$.

Proof. If a fuzzy capacity game $v \in G(N, \widetilde{v})$ is convex, then by the property of the concave integral, $\int^{\mathrm{Ch}} s d v=\int^{\mathrm{Cav}} s d v$ and $\int^{\mathrm{Ch}} u d v=\int^{\mathrm{Cav}} u d v$, so when $v^{\mathrm{Ch}}(\widetilde{S}) \geq v^{\mathrm{Ch}}(\widetilde{U})$, it implies that $v^{\mathrm{Cav}}(\widetilde{S}) \geq v^{\mathrm{Cav}}(\widetilde{U})$.

Conversely, if $v$ is not convex, then there exits a nonnegative variable $x$ such that $\int^{\mathrm{Ch}} x d v \neq \int^{\mathrm{Cav}} x d v$. Since $\int^{\mathrm{Cav}} x d v \geq \int^{\mathrm{Ch}} x d v$, then $\int^{\mathrm{Cav}} x d v>\int^{\mathrm{Ch}} x d v$; there is at least a crisp coalition $S \subseteq N$ and a nonnegative constant $c$ such that

$$
\int^{\mathrm{Cav}} s d v>\int^{\mathrm{Cav}} c 1_{T} d v>\int^{\mathrm{Ch}} s d v
$$

We have

$$
v^{\mathrm{Cav}}(\widetilde{S})>c v(S)>v^{\mathrm{Ch}}(\widetilde{S})
$$

Similarly,

$$
v^{\mathrm{Cav}}(\widetilde{U})>m v(U)>v^{\mathrm{Ch}}(\widetilde{U}),
$$

where the crisp coalition $U \subseteq N$ and $m$ is a nonnegative constant.

Therefore, whenever $v^{\mathrm{Ch}}(\widetilde{S}) \geq v^{\mathrm{Ch}}(\widetilde{U})$, it cannot be confirmed that $v^{\mathrm{Cav}}(\widetilde{S}) \geq v^{\mathrm{Cav}}(\widetilde{U})$; thus $v$ is convex.

Since $\int^{\mathrm{Ch}} X d \mu=\int^{\mathrm{Cav}} X d \mu$ if and only if $\mu$ is convex, conversely $v$ is convex if and only if $v^{\mathrm{Cav}}(\widetilde{S})=v^{\mathrm{Ch}}(\widetilde{S})$, for any $\widetilde{S} \subseteq L(N)$. 


\section{The Shapley Values on Fuzzy Coalition Games with the Concave Integral}

The fuzzy Shapley value is one of the important solutions for fuzzy games. It is interesting to study the Shapley function for game $v \in G^{\mathrm{Cav}}(N)$.

4.1. The Shapley Axioms for Games with Fuzzy Coalitions. Tsurumi et al. defined the Shapley function which is based on the natural extension of carrier and null player to fuzzy games. Before introducing the definition, we provide some notations introduced by Tsurumi et al.

For any $\widetilde{U} \in L(N), \widetilde{S} \in L(\widetilde{U})$, and $i, j \in N$,

$$
\begin{gathered}
\widetilde{S}_{i}^{\widetilde{U}}(j)= \begin{cases}u_{i}, & \text { if } j=i, \\
s_{j}, & \text { otherwise, }\end{cases} \\
\widetilde{S}_{i j}^{\widetilde{U}}(k)= \begin{cases}\min \left\{s_{i}, u_{j}\right\}, & \text { if } k=i, \\
\min \left\{s_{j}, u_{i}\right\}, & \text { if } k=j, \\
s_{k}, & \text { otherwise, }\end{cases} \\
p_{i j}(\widetilde{S})(k)= \begin{cases}s_{j}, & \text { if } k=i, \\
s_{i}, & \text { if } k=j, \\
s_{k}, & \text { otherwise. }\end{cases}
\end{gathered}
$$

Obviously, $\widetilde{S}_{i}^{\widetilde{U}}, \widetilde{S_{i j}}$, and $p_{i j}\left(\widetilde{S_{i j}}\right) \in \widetilde{L}(\widetilde{U})$

Definition 20. Let $G^{\prime}(N, \widetilde{v}) \subseteq G(N, \widetilde{v})$; a function $f$ : $G^{\prime}(N, \widetilde{v}) \rightarrow\left(R_{+}^{n} \cup 0\right)^{L(N)}$ is said to be a Shapley value on $G^{\prime}(N, \widetilde{v})$ if it satisfies the following four axioms.

Axiom 1. If $v \in G^{\prime}(N, \widetilde{v})$ and $\widetilde{U} \in L(N)$, then

$$
\begin{aligned}
& \sum_{i \in \operatorname{Supp} \widetilde{U}} f_{i}(v)(\widetilde{U})=v(\widetilde{U}), \\
& f_{i}(v)(\widetilde{U})=0, \quad \forall i \notin \operatorname{Supp} \widetilde{U},
\end{aligned}
$$

where $f_{i}(v)(\widetilde{U})$ is the ith element of $f(v)(\widetilde{U}) \in R_{+}^{n}$.

Axiom 2. If $v \in G^{\prime}(N, \widetilde{v}), \widetilde{U} \in L(N)$, and $\widetilde{T} \in C(\widetilde{U} \mid v)$, then

$$
f_{i}(v)(\widetilde{U})=f_{i}(v)(\widetilde{T}), \forall i \in N
$$

Axiom 3. If $v \in G^{\prime}(N, \widetilde{v}), \widetilde{U} \in L(N), i, j \in N, \widetilde{U}_{i j}^{\widetilde{U}} \in C(\widetilde{U} \mid v)$, and $v(\widetilde{S})=v\left(p_{i j}[\widetilde{S}]\right)$ for any $\widetilde{S} \in L\left(\widetilde{U}_{i j}^{\widetilde{U}}\right)$, then

$$
f_{i}(v)(\widetilde{U})=f_{j}(v)(\widetilde{U}) .
$$

Axiom 4. For any $\widetilde{v}_{1}, \widetilde{v}_{2} \in G^{\prime}(N, \widetilde{v})$, define a game $v_{1}+v_{2}$ by $\left(v_{1}+v_{2}\right)(\widetilde{S})=v_{1}(\widetilde{S})+v_{2}(\widetilde{S})$ for any $\widetilde{S} \in \widetilde{L}(N)$. If $v_{1}+v_{2} \in$ $G^{\prime}(N, \widetilde{v})$ and $\widetilde{U} \in \widetilde{L}(N)$, then

$$
f_{i}\left(v_{1}+v_{2}\right)(\widetilde{U})=f_{i}\left(v_{1}\right)(\widetilde{U})+f_{i}\left(v_{2}\right)(\widetilde{U}), \quad \forall i \in N
$$

These axioms for the Shapley value are extensions of the crisp Shapley axioms and are suitable to games with fuzzy coalitions. It is unnecessary to transform the Shapley axioms to deal with our fuzzy cooperative games.

Theorem 21. If $v \in v^{C h}(N)$ and $\widetilde{S} \subseteq \widetilde{U} \in L(N)$, then $f_{i}(v)(\widetilde{S}) \leq f_{i}(v)(\widetilde{U}), \forall i \in N$.

Proof. For $\widetilde{S} \subseteq \widetilde{U} \in L(N)$, note that $\widetilde{S} \subseteq \widetilde{U}$ if and only if $[\widetilde{S}]_{h} \subseteq$ $[\widetilde{U}]_{h}$ for any $h \in(0,1]$. Since $v \in G_{0}(N, v)$ is convex, $[\widetilde{S}]_{h} \subseteq$ $[\widetilde{U}]_{h}$ implies that $f_{i}^{\prime}(v)\left([S]_{h}\right) \leq f_{i}^{\prime}(v)\left([T]_{h}\right)$ for any $i \in N$; therefore, $f_{i}(v)(\widetilde{S}) \leq f_{i}(v)(\widetilde{U})$ for any $i \in N$.

4.2. The Shapley Values for Simple Game with Fuzzy Coalitions. Following Shapley [1], with any nonempty coalition $S \in$ $P(N)$, we consider the fuzzy simple game $1_{\widetilde{T}}$ defined by

$$
1_{\widetilde{T}}(A)= \begin{cases}1, & \text { if } \widetilde{T} \subseteq L(A) \\ 0, & \text { otherwise }\end{cases}
$$

and the number

$$
c_{\widetilde{T}}(v)=\sum_{B \in P(N): B \subseteq T}(-1)^{|T|-|B|} v^{\mathrm{Cav}}\left(\sum_{j \in B} t_{j} e^{j}\right),
$$

where $t=\left(t_{1}, t_{2}, \ldots, t_{n}\right)$ is a fuzzy coalition variable of $\widetilde{T} \subseteq$ $L(A)$.

Consider

$$
\begin{aligned}
c_{\widetilde{T}}(v)= & \sum_{B \in P(N): B \subseteq T}(-1)^{|T|-|B|} v^{\mathrm{Cav}}\left(\sum_{j \in B} t_{j} e^{j}\right) \\
= & \sum_{B \in P(N): B \subseteq T}(-1)^{|T|-|B|} \max \left\{\sum_{j \in B} \alpha_{T_{j}} v\left(T_{j}\right),\right. \\
& \left.\sum_{j \in B} \alpha_{T_{j}} 1_{T_{j}}=t, \alpha_{T_{j}} \geq 0\right\}
\end{aligned}
$$

$$
=\max \left\{\sum_{j \in B, B \subseteq T} \alpha_{T_{j}} \sum_{B \in P(N): B \subseteq T}(-1)^{|T|-|B|} v\left(T_{j}\right),\right.
$$$$
\left.\sum_{j \in B} \alpha_{T_{j}} 1_{T_{j}}=t, \alpha_{T_{j}} \geq 0\right\}
$$$$
=\max \left\{\sum_{j \in B, B \subseteq T} \alpha_{T_{j}} c_{T_{j}}(v),\right.
$$

$$
\left.\sum_{j \in B} \alpha_{T_{j}} 1_{T_{j}}=t, \alpha_{T_{j}} \geq 0\right\} .
$$


In addition, for any a cooperative game $v \in G(N, v)$,

$$
\begin{aligned}
& \sum_{\widetilde{T} \in L(N) \backslash\{\phi\}} c_{\widetilde{T}}\left(v^{\text {Cav }}\right) 1_{\widetilde{T}} \\
& =\sum_{\widetilde{T} \in L(N) \backslash\{\phi\}} \max \left\{\sum_{j \in B, B \subseteq T} \alpha_{T_{j}} c_{T_{j}}(v),\right. \\
& \left.\sum_{j \in B} \alpha_{T_{j}} 1_{T_{j}}=t, \alpha_{T_{j}} \geq 0\right\} 1_{\widetilde{T}} \\
& =\max \sum_{\tilde{T} \in L(N) \backslash\{\phi\}}\left\{\sum_{j \in B, B \subseteq T} \alpha_{T_{j}} c_{T_{j}}(v),\right. \\
& \left.\sum_{j \in B} \alpha_{T_{j}} 1_{T_{j}}=t, \alpha_{T_{j}} \geq 0\right\} 1_{\widetilde{T}} \\
& =\max \sum_{\widetilde{T} \in L(N) \backslash\{\phi\}} \sum_{j \in B, B \subseteq T} \alpha_{T_{j}} c_{T_{j}}(v) 1_{\widetilde{T}}, \\
& \left(\sum_{j \in B} \alpha_{T_{j}} 1_{T_{j}}=t, \alpha_{T_{j}} \geq 0\right) \\
& =\max \sum_{j \in B, B \subseteq T} \alpha_{T_{j}} \sum_{\widetilde{T} \in L(N) \backslash\{\phi\}} c_{T_{j}}(v) 1_{\widetilde{T}}, \\
& \left(\sum_{j \in B} \alpha_{T_{j}} 1_{T_{j}}=t, \alpha_{T_{j}} \geq 0\right) \\
& =\max \sum_{j \in B, B \subseteq T} \alpha_{T_{j}} \sum_{\operatorname{supp}(\widetilde{T}) \in P(N) \backslash\{\phi\}} c_{T_{j}}(v) 1_{\operatorname{supp}(\widetilde{T})}, \\
& \left(\sum_{j \in B} \alpha_{T_{j}} 1_{T_{j}}=t, \alpha_{T_{j}} \geq 0\right) \\
& =\max \sum_{j \in B, B \subseteq T} \alpha_{T_{j}} \sum_{\operatorname{supp}(\widetilde{T}) \in P(N) \backslash\{\phi\}} c_{T_{j}}(v) 1_{\text {supp }(\widetilde{T})}, \\
& \left(\sum_{j \in B} \alpha_{T_{j}} 1_{T_{j}}=t, \alpha_{T_{j}} \geq 0\right) \\
& =\max \sum_{j \in B, B \subseteq T} \alpha_{T_{j}} v\left(T_{j}\right), \\
& \left(\sum_{j \in B} \alpha_{T_{j}} 1_{T_{j}}=t, \alpha_{T_{j}} \geq 0\right) \\
& =v^{\mathrm{Cav}}(\widetilde{T}) \text {. }
\end{aligned}
$$

Hence,

$$
v^{\mathrm{Cav}}(\widetilde{T})=\sum_{\widetilde{T} \in L(N) \backslash\{\phi\}} c_{\widetilde{T}}\left(v^{\mathrm{Cav}}\right) 1_{\widetilde{T}} .
$$

Lemma 22. Let $\widetilde{S} \in L(N)$; then for any $\widetilde{T} \subseteq L(\widetilde{S})$, a Shapley value on the simple game $1_{\widetilde{T}}$ is as follows:

$$
f_{i}\left(1_{\widetilde{T}}\right)(\widetilde{S})= \begin{cases}\frac{1}{|\widetilde{T}|}, & \text { if } i \in \operatorname{Supp}(\widetilde{T}), \\ 0, & \text { otherwise. }\end{cases}
$$

Proof. Let $\widetilde{S} \in L(N)$ with fuzzy coalition variable $s=$ $\left(s_{1}, s_{2}, \ldots, s_{n}\right)$; for all $\widetilde{T} \subseteq L(\widetilde{S})$, it is obvious that $1_{\widetilde{T}}(\widetilde{S} \cap \widetilde{T})=$ $1_{\widetilde{T}}(\widetilde{T})$, and $\widetilde{T}$ is a fuzzy carrier in fuzzy coalition $\widetilde{S}$.

Axiom 1. Consider

$$
\begin{gathered}
\sum_{i \in \operatorname{Supp}(\widetilde{S})} f_{i}\left(1_{\widetilde{T}}\right)(\widetilde{S})=1_{\widetilde{T}}(\widetilde{S})=1_{\widetilde{T}}(\widetilde{T}) \\
=\sum_{i \in \operatorname{Supp}(\widetilde{T})} \frac{1}{|\widetilde{T}|}=\sum_{i \in \operatorname{Supp}(\widetilde{T})} f_{i}\left(1_{\widetilde{T}}\right)(\widetilde{T}), \\
\sum_{i \notin \operatorname{Supp}(\widetilde{S})} f_{i}\left(1_{\widetilde{T}}\right)(\widetilde{S})=0 .
\end{gathered}
$$

That is,

$$
\begin{gathered}
\sum_{i \in \operatorname{Supp}(\widetilde{S})} f_{i}\left(1_{\widetilde{T}}\right)(\widetilde{S})=1_{\widetilde{T}}(\widetilde{S}), \\
f_{i}\left(1_{\widetilde{T}}\right)(\widetilde{S})=0, \quad \forall i \notin \operatorname{Supp} \widetilde{S} .
\end{gathered}
$$

Axiom 2. For any $k \in \operatorname{Supp}(\widetilde{S}) \backslash \operatorname{Supp}(\widetilde{T}), f_{k}\left(1_{\widetilde{T}}\right)(\widetilde{S})=0 . \widetilde{T} \subseteq$ $\widetilde{T} \cup\left(s_{k} e^{k}\right)$ and $1_{\widetilde{T}}\left(\widetilde{T} \cup\left(s_{k} e^{k}\right)\right)=1_{\widetilde{T}}(\widetilde{T})$; then $\widetilde{T} \cup\left(s_{k} e^{k}\right)$ is also a fuzzy carrier in coalition $\widetilde{S}$, so

$$
f_{k}\left(1_{\widetilde{T}}\right)(\widetilde{S})=f_{k}\left(1_{\widetilde{T}}\right)(\widetilde{T}) .
$$

For any $k \in \operatorname{Supp}(\widetilde{T}), f_{k}\left(1_{\widetilde{T}}\right)(\widetilde{S})=1 /|\widetilde{T}|$ and $f_{k}\left(1_{\widetilde{T}}\right)(\widetilde{T})=$ $1 /|\widetilde{T}|$.

Axiom 3. For any $i, j \in \operatorname{Supp} \widetilde{T}$, let $\widetilde{B} \in L(\operatorname{Supp} \widetilde{S} \backslash\{i, j\})$; by the fuzzy simple definition, $1_{\widetilde{T}}\left(\widetilde{B} \cup\left(s_{i} e^{i}\right)\right)=1_{\widetilde{T}}\left(\widetilde{B} \cup\left(s_{j} e^{j}\right)\right)=0$, so $f_{i}\left(1_{\widetilde{T}}\right)(\widetilde{S})=f_{j}\left(1_{\widetilde{T}}\right)(\widetilde{S})$.

Axiom 4. For any two fuzzy simple games $1_{\widetilde{T}_{1}}$ and $1_{\widetilde{T}_{2}}$, define $\left(1_{\widetilde{T}_{1}}+1_{\widetilde{T}_{2}}\right)(\widetilde{S})=1_{\widetilde{T}_{1}}(\widetilde{S})+1_{\widetilde{T}_{2}}(\widetilde{S}), \forall \widetilde{S} \in L(N)$. If $1_{\widetilde{T}_{1}}+1_{\widetilde{T}_{2}}$ is also a fuzzy simple game, then

$$
\begin{aligned}
\sum_{i} f_{i}\left(1_{\widetilde{T}_{1}}+1_{\widetilde{T}_{2}}\right)(\widetilde{S}) & =\left(1_{\widetilde{T}_{1}}+1_{\widetilde{T}_{2}}\right)(\widetilde{S})=1_{\widetilde{T}_{1}}(\widetilde{S})+1_{\widetilde{T}_{2}}(\widetilde{S}) \\
& =\sum_{i} f_{i}\left(1_{\widetilde{T}_{1}}\right)(\widetilde{S})+\sum_{i} f_{i}\left(1_{\widetilde{T}_{2}}\right)(\widetilde{S}) .
\end{aligned}
$$

It satisfies for any $i \in N, f_{i}\left(1_{\widetilde{T}_{1}}+1_{\widetilde{T}_{2}}\right)(\widetilde{S})=f_{i}\left(1_{\widetilde{T}_{1}}\right)(\widetilde{S})+$ $f_{i}\left(1_{\widetilde{T}_{2}}\right)(\widetilde{S})$.

It is evident that $f_{i}\left(1_{\widetilde{T}}\right)(\widetilde{S})=f_{i}\left(1_{\widetilde{T}}\right)(\widetilde{T}), \forall i \in N$. 
4.3. The Shapley Values for Game with Concave Integral. For a fuzzy coalition $\widetilde{S} \in L(N)$ with fuzzy coalition variable $s=$ $\left(s_{1}, s_{2}, \ldots, s_{n}\right)$, we simply denote it by crisp game marks as follows:

$$
\begin{gathered}
w_{\widetilde{S}}(\{1\})=v^{\operatorname{Cav}}\left(s_{1} e^{1}\right), \\
w_{\widetilde{S}}(\{1,2\})=v^{\operatorname{Cav}}\left(\sum_{j=1}^{2} s_{j} e^{j}\right), \\
\vdots \\
w_{\widetilde{S}}(T)=v^{\operatorname{Cav}}\left(\sum_{j \in T} s_{j} e^{j}\right), \\
\vdots \\
w_{\widetilde{S}}(N)=v^{\operatorname{Cav}}\left(\sum_{j \in N} s_{j} e^{j}\right) .
\end{gathered}
$$

If we let $c_{\widetilde{T}}(w)=\sum_{B \in L(N): B \subseteq S u p p \widetilde{T}}(-1)^{|\widetilde{T}|-|B|} w_{\widetilde{S}}(B)$, it is obvious that $c_{\widetilde{T}}(v)$ is a linear sum with the concave integral on $L(\widetilde{S})$, and

$$
\begin{aligned}
& \sum_{\widetilde{T} \in L(N) \backslash\{\phi\}} c_{\widetilde{T}}(w) 1_{\widetilde{T}}(B) \\
& \quad=\sum_{\widetilde{T} \in L(N) \backslash\{\phi\}} \sum_{B \in L(N): B \subseteq S \sup \widetilde{T}}(-1)^{|\widetilde{T}|-|B|} w_{\widetilde{S}}(B) 1_{\widetilde{T}}(B) \\
& \quad=w_{\widetilde{S}}(B) .
\end{aligned}
$$

Therefore,

$$
\begin{aligned}
f_{i}\left(w_{\widetilde{S}}\right)(\widetilde{S}) & =f_{i}\left(\sum_{\widetilde{T} \in L(N) \backslash\{\phi\}} c_{\widetilde{T}}(w) 1_{\widetilde{T}}(B)\right)(\widetilde{S}) \\
& =\sum_{\widetilde{T} \in L(N) \backslash\{\phi\}} c_{\widetilde{T}}(w) f_{i}\left(1_{\widetilde{T}}(B)\right)(\widetilde{S}) \\
& =\sum_{\widetilde{T} \in L(N) \backslash\{\phi\}} c_{\widetilde{T}}(w) \frac{1}{|\widetilde{T}|} \\
& =\sum_{\widetilde{T} \in L(N) \backslash\{\phi\}} \sum_{i \in T, B \in P(N): B \subseteq T}(-1)^{|T|-|B|} w_{\widetilde{S}}(B) \frac{1}{|T|} \\
& =\sum_{i \in T \subseteq S \in P(N)} \beta(|T| ;|S|) \cdot\left\{w_{\widetilde{S}}(T)-w_{\widetilde{S}}(T \backslash i)\right\},
\end{aligned}
$$

where $T=\sum_{j \in T} s_{j} e^{j}$ and $S=\sum_{j \in S} s_{j} e^{j}$.
Hence, the Shapley value of player $i$ on the fuzzy coalition $\widetilde{S}$ will be

$$
\begin{aligned}
f_{i}\left(w_{\widetilde{S}}\right)(\widetilde{S})= & \sum_{i \in T \subseteq S \in P(N)} \frac{(|T|-1) !(|S|-|T|) !}{|S| !} \\
& \times\left\{w_{\widetilde{S}}(T)-w_{\widetilde{S}}(T \backslash i)\right\} .
\end{aligned}
$$

Lemma 23. Let $v \in G^{C a v}(N)$ be a fuzzy capacity game, and let $\widetilde{S} \in L(N)$ be a random fuzzy coalition with nonnegative variable $s=\left(s_{1}, s_{2}, \ldots, s_{n}\right)$; then the vector

$$
f\left(w_{\widetilde{S}}\right)(\widetilde{S})=\left(f_{1}\left(w_{\widetilde{S}}\right)(\widetilde{S}), f_{2}\left(w_{\widetilde{S}}\right)(\widetilde{S}), \ldots, f_{n}\left(w_{\widetilde{S}}\right)(\widetilde{S})\right)
$$

is an imputation of the fuzzy coalition $\widetilde{S}$, where $f_{i}\left(w_{\widetilde{S}}\right)(\widetilde{S})$ is defined as (65).

Proof. For any $i \notin \operatorname{supp} \widetilde{S}$, it is apparent that $f_{i}\left(w_{\widetilde{S}}\right)(\widetilde{S})=0$ and $\sum_{i \in \operatorname{supp} \widetilde{S}} f_{i}\left(w_{\widetilde{S}}\right)(\widetilde{S})=w_{\widetilde{S}}(S)$. So $\sum_{i \in N} f_{i}\left(w_{\widetilde{S}}\right)(\widetilde{S})=w_{\widetilde{S}}(S)$.

Meanwhile,

$$
\begin{aligned}
f_{i}\left(w_{\widetilde{S}}\right)(\widetilde{S})= & \sum_{i \in T \subseteq S \in P(N)} \frac{(|T|-1) !(|S|-|T|) !}{|S| !} \\
& \times\left\{w_{\widetilde{S}}(T)-w_{\widetilde{S}}(T \backslash i)\right\} \\
\geq & \sum_{i \in T \subseteq S \in P(N)} \frac{(|T|-1) !(|S|-|T|) !}{|S| !} w_{\widetilde{S}}(i) \\
= & w_{\widetilde{S}}(i)=v^{\mathrm{Cav}}\left(s_{i} e^{i}\right) \geq s_{i} v\left(e^{i}\right) .
\end{aligned}
$$

Therefore, we have $f_{i}\left(w_{\widetilde{S}}\right)(\widetilde{S}) \geq s_{i} v\left(\left\{e^{i}\right\}\right)$ for any $i \in \widetilde{S}$, so $f\left(w_{\widetilde{S}}\right)(\widetilde{S})$ is an imputation.

Lemma 24. Let $v \in G^{C a v}(N)$ be a convex game; for any fuzzy coalitions $\widetilde{S}, \widetilde{U} \in L(N)$ such that $\widetilde{S} \subseteq \widetilde{U}$; then $f_{i}\left(w_{\widetilde{S}}\right)(\widetilde{S}) \leq$ $f_{i}\left(w_{\widetilde{U}}\right)(\widetilde{U})(\forall i \in N)$.

Proof. If $v \in G^{\mathrm{Cav}}(N)$ is convex implies that $v \in G^{\mathrm{Ch}}(N)$. From Theorem 21, for fuzzy coalitions $\widetilde{S}, \widetilde{U} \in L(N)$, then $f_{i}(v)(\widetilde{S}) \leq f_{i}(v)(\widetilde{U})$ for any $i \in N$.

Therefore, for any $i \in N, f_{i}\left(w_{\widetilde{S}}\right)(\widetilde{S}) \leq f_{i}\left(w_{\widetilde{S}}\right)(\widetilde{U}) \leq$ $f_{i}\left(w_{\widetilde{U}}\right)(\widetilde{U})$.

Lemma 24 suggests that on game $v \in G^{\mathrm{Cav}}(N), f_{i}\left(w_{\widetilde{S}}\right)(\widetilde{S})$ is monotone nondecreasing with respect to fuzzy coalition variable when $v \in G^{\mathrm{Cav}}(N)$ is convex. In fact, $f_{i}\left(w_{\widetilde{S}}\right)(\widetilde{S})$ is also continuous with respect to fuzzy coalition variable when $v \in G^{\text {Cav }}(N)$.

The next lemma can easily be proved by the same manner as Lemma 24.

Lemma 25. Let $v \in G^{C a v}(N)$; then $f_{i}\left(w_{\widetilde{S}}\right)(\widetilde{S})$ is also continuous with respect to fuzzy coalition variable for any $i \in N$.

Example 26. Let $N=\{1,2,3,4\}, \widetilde{S} \in L(N)$ with the fuzzy coalition variable $s=\left(s_{1}, 0.4,0.7,0.7\right), v(1)=120, v(2)=150$, 
$v(3)=v(4)=180, v(1,2)=450, v(1,3)=v(1,4)=v(2,3)=$ $v(2,4)=480, v(3,4)=600, v(1,2,3)=v(1,2,4)=840$, $v(2,3,4)=v(1,3,4)=900, v(1,2,3,4)=1500$.

Now we consider the Shapley value of player 1 in fuzzy coalition $\widetilde{S}$ by $(65)$; we have

$$
f_{1}\left(w_{\widetilde{S}}\right)(\widetilde{S})= \begin{cases}340 s_{1}, & 0 \leq s_{1}<0.4 \\ 240 s_{1}+40, & 0 \leq s_{1}<0.4 \\ 120 s_{1}+124, & 0 \leq s_{1}<0.4\end{cases}
$$

Note that the game is convex such that the Shapley $f_{1}\left(w_{\widetilde{S}}\right)(\widetilde{S})$ is the same as that of Tsurumi game.

Theorem 27. Let $v \in G^{C a v}(N)$ be a fuzzy capacity game, and let $\widetilde{S} \in L(N)$ be a random fuzzy coalition with nonnegative variable $s=\left(s_{1}, s_{2}, \ldots, s_{n}\right)$; reorder its components such that $s_{j_{1}}<s_{j_{2}}<\cdots<s_{j_{n}}$; then $f_{i}\left(w_{\widetilde{S}}\right)(\widetilde{S})(\forall i \in N)$ can be calculated by

$$
\begin{aligned}
& f_{i}\left(w_{\widetilde{S}}\right)(\widetilde{S}) \\
& =\max \sum_{i \in T \subseteq S \in P(N)} \frac{(|T|-1) !(|S|-|T|) !}{|S| !} \\
& \quad \times\left\{\left\{v\left(T \cap T_{1}\right)-v\left((T \backslash i) \cap T_{1}\right)\right\} \alpha_{s_{j_{1}}}\right. \\
& \quad+\left\{v\left(T \cap T_{2}\right)-v\left((T \backslash i) \cap T_{2}\right)\right\} \alpha_{s_{j_{2}}} \\
& \left.+\cdots+\left\{v\left(T \cap T_{m}\right)-v\left((T \backslash i) \cap T_{m}\right)\right\} \alpha_{s_{j_{m}}}\right\},
\end{aligned}
$$

where $\sum_{S_{j}} \alpha_{s_{j}} 1_{S_{j}} \leq s$ and $s_{j_{m}}=s_{i}(1 \leq m \leq n)$.

Proof. Let $Q(T)=Q\left(\sum_{j \in T} s_{j} e^{j}\right)=\left\{s_{j} \mid s_{j}>0, j \in T\right\}, q=$ $|Q(T)|$; reorder the elements in $Q(T)$ such that $s_{j_{1}}<s_{j_{2}}<$ $\cdots<s_{j_{q}}$.

For the fuzzy variable $s$, let $\sum_{j=1}^{q} \alpha_{s_{j}} 1_{S_{j}} \leq s$, where $\alpha_{s_{j}}=1$ if the set $T_{j}$ is a subdecomposition set, else $\alpha_{s_{j}}=0$ and $S_{j} \in N$ $(j=1,2, \ldots, q)$. Then all the subdecomposition sets $S_{1}<S_{2}<$ $\cdots<S_{q}$ correspond with the order.

Let $s_{j_{m}}=s_{i}, 1 \leq m \leq q$. For any a subdecomposition $T$, by $v \in G^{\mathrm{Cav}}(N)$,

$$
\begin{gathered}
v\left(\sum_{j \in T} s_{j} e^{j}\right)-v\left(\sum_{j \in T \backslash i} s_{j} e^{j}\right) \\
=w_{\widetilde{S}}(T)-w_{\widetilde{S}}(T \backslash i) \\
=\max \left\{v\left(T \cap T_{1}\right) \alpha_{s_{j_{1}}}+v\left(T \cap T_{2}\right) \alpha_{s_{j_{2}}}\right. \\
\left.+\cdots+v\left(T \cap T_{q}\right) \alpha_{s_{j_{q}}}\right\} \\
-\max \left\{v\left((T \backslash i) \cap T_{1}\right) \alpha_{s_{j_{1}}}-v\left((T \backslash i) \cap T_{2}\right) \alpha_{s_{j_{2}}}\right. \\
\left.-\cdots-v\left((T \backslash i) \cap T_{q}\right) \alpha_{s_{j_{q}}}\right\}
\end{gathered}
$$

It is obvious that $\left(T \cap T_{k}\right)=v\left((T \backslash i) \cap T_{k}\right)$; for any $m+1 \leq$ $k<q$, we get

$$
\begin{aligned}
& w_{\widetilde{S}}(T)-w_{\widetilde{S}}(T \backslash i) \\
&=\max \left\{v\left(T \cap T_{1}\right) \alpha_{s_{j_{1}}}+v\left(T \cap T_{2}\right) \alpha_{s_{j_{2}}}\right. \\
&\left.+\cdots+v\left(T \cap T_{m}\right) \alpha_{s_{j_{m}}}\right\} \\
&-\max \left\{v\left((T \backslash i) \cap T_{1}\right) \alpha_{s_{j_{1}}}\right. \\
& \quad-v\left((T \backslash i) \cap T_{2}\right) \alpha_{s_{j_{2}}} \\
&\left.-\cdots-v\left((T \backslash i) \cap T_{m}\right) \alpha_{s_{j_{m}}}\right\} .
\end{aligned}
$$

Therefore,

$$
\begin{aligned}
& f_{i}\left(w_{\widetilde{S}}\right)(\widetilde{S})=\sum_{i \in T \subseteq S \in P(N)} \frac{(|T|-1) !(|S|-|T|) !}{|S| !} \\
& \times\left\{w_{\widetilde{S}}(T)-w_{\widetilde{S}}(T \backslash i)\right\} \\
& =\sum_{i \in T \subseteq S \in P(N)} \frac{(|T|-1) !(|S|-|T|) !}{|S| !} \\
& \times \sum_{j} \max \left\{v\left(T \cap T_{j}\right)-v\left((T \backslash i) \cap T_{j}\right)\right\} \alpha_{s_{j}} \\
& =\max \sum_{i \in T \subseteq S \in P(N)} \frac{(|T|-1) !(|S|-|T|) !}{|S| !} \\
& \times\left\{\left\{v\left(T \cap T_{1}\right)\right.\right. \\
& \left.-v\left((T \backslash i) \cap T_{1}\right)\right\} \alpha_{s_{j_{1}}} \\
& +\left\{v\left(T \cap T_{2}\right)-v\left((T \backslash i) \cap T_{2}\right)\right\} \alpha_{s_{j_{2}}} \\
& +\cdots+\left\{v\left(T \cap T_{m}\right)\right. \\
& \left.\left.-v\left((T \backslash i) \cap T_{m}\right)\right\} \alpha_{s_{j_{m}}}\right\} \text {. }
\end{aligned}
$$

Define a function $f: G^{\text {Cav }}(N) \rightarrow\left(R_{+}^{n} \cup\{0\}\right)^{L(N)}$ by

$$
\begin{array}{ll}
f_{i}(v)(\widetilde{S})=\max & \sum_{T_{j} \subseteq N} \alpha_{T_{j}} f_{i}^{\prime}(v)\left(T_{j}\right) \\
\text { s.t. } \quad & \sum_{T_{j}} \alpha_{T_{j}} 1_{T_{j}}=s, \\
& T_{j} \subseteq N, \quad\left(j=1,2, \ldots, 2^{n}\right), \\
& \alpha_{T_{j}} \geq 0, \quad\left(j=1,2, \ldots, 2^{n}\right),
\end{array}
$$

where $f^{\prime}$ is the function given in Theorem 4 .

Note that (73) is a concave integral on the fuzzy coalition $\widetilde{S}$ with regard to $f^{\prime}(v)$. Next we will show that $f$ is a Shapley function on $G^{\text {Cav }}(N)$. 
Theorem 28. Let $v \in G^{C a v}(N)$ be a fuzzy capacity game, and let $\widetilde{S} \in L(N)$ be a random fuzzy coalition with nonnegative variable $s=\left(s_{1}, s_{2}, \ldots, s_{n}\right)$; then the function defined by $(73)$ is a Shapley function on $G^{\mathrm{Cav}}(N)$.

Proof. We will show that $f$ satisfies the axioms in Definition 20.

Axiom 1. Let $v \in G^{\mathrm{Cav}}(N)$ and $\widetilde{S} \in L(N)$. By $\sum_{i \in N} f_{i}^{\prime}(v)\left(T_{j}\right)=$ $v\left(T_{j}\right)$ for any $T_{j} \subseteq N$, we get

$$
\begin{aligned}
\sum_{i \in \operatorname{Supp} \tilde{S}} f_{i}(v)(\widetilde{S})= & \sum_{i \in \operatorname{Supp} \widetilde{S}} \max \sum_{T_{j} \subseteq N} \alpha_{T_{j}} f_{i}^{\prime}(v)\left(T_{j}\right) \\
& =\max \sum_{T_{j} \subseteq N} \alpha_{T_{j}} \sum_{i \in \operatorname{Supp}} f_{i}^{\prime}(v)\left(T_{j}\right) \\
& =\max \sum_{T_{j} \subseteq N} \alpha_{T_{j}} v\left(T_{j}\right)=v^{\mathrm{Cav}}(\widetilde{S})
\end{aligned}
$$

$$
\begin{array}{ll}
\text { s.t. } & \sum_{T_{j}} \alpha_{T_{j}} 1_{T_{j}}=s, \\
& T_{j} \subseteq N, \quad\left(j=1,2, \ldots, 2^{n}\right), \\
& \alpha_{T_{j}} \geq 0, \quad\left(j=1,2, \ldots, 2^{n}\right) .
\end{array}
$$

If $i \notin \operatorname{Supp} \widetilde{S}$, then $i \notin T_{j}(j=1,2, \ldots, n)$ such that $f_{i}^{\prime}(v)\left(T_{j}\right)=$ 0 . So $f_{i}(v)(\widetilde{S})=0$ when $i \notin \operatorname{Supp} \widetilde{S}$.

Thus,

$$
\begin{gathered}
\sum_{i \in \operatorname{Supp}} f_{i}(v)(\widetilde{S})=v(\widetilde{S}), \\
f_{i}(v)(\widetilde{S})=0, \quad \forall i \notin \operatorname{Supp} \widetilde{S} .
\end{gathered}
$$

Axiom 2. Let $\widetilde{S} \in L(N)$; denote a subdecomposition $\bar{S}=$ $\left\{\sum_{j} \alpha_{j} S_{j} \mid S_{j} \in P(N), j \in 2^{n}\right\}$ such that $\sum_{j} \alpha_{j} 1_{S_{j}} \leq s$ and $\sum_{j} \alpha_{s_{j}}=1$. Let $\widetilde{T} \in C(\widetilde{S} \mid v)$; then its subdecomposition is denoted by $\bar{T}$; this implies that $\bar{T} \in C(\bar{S} \mid v)$, so $f_{i}^{\prime}(v)(\bar{S})=$ $f_{i}^{\prime}(v)(\bar{T})$. We can easily obtain

$$
f_{i}(v)(\widetilde{S})=f_{i}(v)(\widetilde{T}), \quad \forall i \in N .
$$

Axiom 3. Let $\widetilde{S} \in L(N), \widetilde{S}_{i j}^{\widetilde{S}} \in C(\widetilde{S} v)$, and $v(\widetilde{T})=v\left(p_{i j}[\widetilde{T}]\right)$, for any $\widetilde{T} \in L\left(\widetilde{S}_{i j}^{\widetilde{S}}\right)$. We note that $\widetilde{S}_{i j}^{\widetilde{S}}(i)=\widetilde{S}_{i j}^{\widetilde{S}}(j)$; if $i, j \notin \operatorname{Supp} \widetilde{S}_{i j}^{\widetilde{S}}$ then $f_{i}(v)\left(\widetilde{S}_{i j}^{\tilde{S}}\right)=f_{j}(v)\left(\widetilde{S}_{i j}^{\widetilde{S}}\right)=0$.

Since $\widetilde{S}_{i j} \in C(\widetilde{S} \mid v)$, by Axiom 1, we have $f_{i}(v)(\widetilde{S})=$ $f_{j}(v)(\widetilde{S})=f_{i}(v)\left(\widetilde{S}_{i j}^{\widetilde{S}}\right)=f_{j}(v)\left(\widetilde{S}_{i j}^{S}\right)=0$.

On the other hand, if $i, j \in \operatorname{Supp} \widetilde{S} \widetilde{S}$ that means $i, j \in$ Supp $\widetilde{S}$ and $\widetilde{S}=\widetilde{S}_{i j}$.

From $v(\widetilde{T})=v\left(p_{i j}[\widetilde{T}]\right)$ for any $\widetilde{T} \in L\left(\widetilde{S_{i j}}\right)$, we get

$$
v\left(\widetilde{T} \cup e^{j}\right)=v\left(\widetilde{T} \cup e^{i}\right) \quad \text { for } \widetilde{T} \in L(\operatorname{Supp} \widetilde{S} \backslash\{i, j\}) .
$$

So from the proof of Axiom 2, we obtain $f_{i}^{\prime}(v)(\bar{S})=f_{j}^{\prime}(v)(\bar{S})$; then the following equation is found:

$$
\begin{aligned}
f_{i}(v)\left(\widetilde{S}_{i j}^{\widetilde{S}}\right) & =\sum_{i \in \operatorname{Supp}} \tilde{S}_{i j} \max \sum_{T_{j} \subseteq N} \alpha_{T_{j}} f_{i}^{\prime}(v)\left(T_{j}\right) \\
& =\sum_{i \in \bar{S}} \max f_{i}^{\prime}(v)(\bar{S})=\sum_{j \in \bar{S}} \max f_{j}^{\prime}(v)(\bar{S}) \\
& =f_{j}(v)\left(\widetilde{S}_{i j} \tilde{S}\right) .
\end{aligned}
$$

Thus,

$$
f_{i}(v)(\widetilde{S})=f_{j}(v)(\widetilde{S})
$$

Axiom 4. Let $\widetilde{v}_{1}, \widetilde{v}_{2} \in G^{\mathrm{Cav}}(N)$; define a game $v_{1}+v_{2}$ by $\left(v_{1}+\right.$ $\left.v_{2}\right)(\widetilde{U})=v_{1}(\widetilde{U})+v_{2}(\widetilde{U})$ for any $\widetilde{U} \in \widetilde{L}(N)$.

$$
\text { If } v_{1}+v_{2} \in G^{\mathrm{Cav}}(N) \text { and } \widetilde{S} \in L(N) \text {, then }
$$

$$
\begin{aligned}
& \sum_{i \in \operatorname{Supp} \tilde{S}} f_{i}\left(v_{1}+v_{2}\right)(\widetilde{S}) \\
& =\sum_{i \in \operatorname{Supp} \tilde{S}} \max \sum_{T_{j} \subseteq N} \alpha_{T_{j}} f_{i}^{\prime}\left(v_{1}+v_{2}\right)\left(T_{j}\right) \\
& =\sum_{i \in \operatorname{Supp} \tilde{S}} \max \sum_{T_{j} \subseteq N}\left\{\alpha_{T_{j}} f_{i}^{\prime}\left(v_{1}\right)\left(T_{j}\right)+\alpha_{T_{j}} f_{i}^{\prime}\left(v_{2}\right)\left(T_{j}\right)\right\} \\
& =\sum_{i \in \operatorname{Supp} \tilde{S}} f_{i}\left(v_{1}\right)(\widetilde{S})+f_{i}\left(v_{2}\right)(\widetilde{S}) .
\end{aligned}
$$

Therefore,

$$
f_{i}\left(v_{1}+v_{2}\right)(\widetilde{S})=f_{i}\left(v_{1}\right)(\widetilde{S})+f_{i}\left(v_{2}\right)(\widetilde{S}), \quad \forall i \in N .
$$

These axioms for the Shapley value are extensions of the crisp Shapley axioms and are suitable to games with fuzzy coalitions.

Example 29. We recall Example 15; the fuzzy coalition $s=$ $(1,0.4,0.6)$ has the maximum subdecomposition $\sum_{T_{i} \subseteq N} \alpha_{T_{i}} T_{i}$ such that $v^{\mathrm{Cav}}(\widetilde{S})=\sum_{T_{i} \subseteq N} \alpha_{T_{i}} v\left(T_{i}\right)$, where $\alpha_{T_{1}}=0.4, \alpha_{T_{2}}=$ $0.6, T_{1}=\{1,2\}$, and $T_{2}=\{1,3\}$. $T_{2}$ :

First, we calculate the Shapley values on coalition $T_{1}$ and

$$
\begin{aligned}
& f_{1}^{\prime}(v)\left(T_{1}\right)=\frac{1}{2}\left(v\left(T_{1}\right)-v(2)\right)+\frac{1}{2} v(1)=\frac{9}{2}, \\
& f_{2}^{\prime}(v)\left(T_{1}\right)=\frac{1}{2}\left(v\left(T_{1}\right)-v(1)\right)+\frac{1}{2} v(2)=\frac{9}{2} .
\end{aligned}
$$

Similarly, $f_{1}^{\prime}(v)\left(T_{2}\right)=f_{2}^{\prime}(v)\left(T_{2}\right)=4$. 
So from Theorem 28,

$$
\begin{aligned}
f_{1}(v)(\widetilde{S}) & =\alpha_{T_{1}} f_{1}^{\prime}(v)\left(T_{1}\right)+\alpha_{T_{2}} f_{1}^{\prime}(v)\left(T_{2}\right) \\
& =0.4 \times \frac{9}{2}+0.6 \times 4=4.2, \\
f_{2}(v)(\widetilde{S}) & =\alpha_{T_{1}} f_{2}^{\prime}(v)\left(T_{1}\right)+\alpha_{T_{2}} f_{2}^{\prime}(v)\left(T_{2}\right) \\
& =0.4 \times \frac{9}{2}+0.6 \times 4=4.2 .
\end{aligned}
$$

Note that $f_{1}(v)(\widetilde{S})+f_{2}(v)(\widetilde{S})=v^{\mathrm{Cav}}(\widetilde{S})$.

4.4. The Shapley Values on Restricted Fuzzy Coalitions of the Concave Game. For the convenience to give available fuzzy imputation on restrict fuzzy coalitions $\widetilde{U}$, let us define $\widetilde{S} \in$ $L(\widetilde{U})$ with the fuzzy coalition variable $s^{u}=\sum_{i \in S} u_{i} e^{i}$.

Definition 30. A function $y: L(\widetilde{U}) \rightarrow R_{+}^{n}$ is said to be imputation for a fuzzy game $v \in G(N, \widetilde{v})$ in fuzzy coalition $\widetilde{U} \in L(N)$ with fuzzy coalition variable $u=\left(u_{1}, u_{2}, \ldots, u_{n}\right)$, if

(1) $y_{i}(\widetilde{U})=0, \forall i \notin \operatorname{Supp}(\widetilde{U})$,

(2) $\sum_{i \in N} y_{i}(\widetilde{U})=v(\widetilde{U})$,

(3) $y_{i}(\widetilde{U}) \geq v\left(i^{\widetilde{U}}\right)$,

where $y_{i}(\widetilde{U})=\left(y_{1}(\widetilde{U}), y_{2}(\widetilde{U}), \ldots, y_{n}(\widetilde{U})\right)$.

The player $i \in N$ is a dummy player on restrict fuzzy coalition $\widetilde{U} \in L(N)$ when

$$
v\left(\widetilde{S}^{\widetilde{U}} \cup i^{\widetilde{U}}\right)=v\left(\widetilde{S}^{\widetilde{U}}\right)+v\left(i^{\widetilde{U}}\right) .
$$

And the player $i$ is a null player if

$$
v\left(\widetilde{S}^{\widetilde{U}} \cup i^{\widetilde{U}}\right)=v(\widetilde{S})
$$

Definition 31. Let $v \in G(N, \widetilde{v}), \widetilde{U} \in L(N)$; then $\widetilde{S} \in L(\widetilde{U})$ is called a fuzzy carrier in restrict coalition $\widetilde{U}$, if for any $\forall \widetilde{T} \in$ $L(\widetilde{U})$ such that

$$
v\left(\widetilde{S}^{\widetilde{U}} \cap \widetilde{T}^{\widetilde{U}}\right)=v\left(\widetilde{T}^{\widetilde{U}}\right)
$$

Denoting the carrier set as $C_{\widetilde{U}}(v)$, it is obvious that

$$
\begin{aligned}
C_{\widetilde{U}} & (v) \\
\quad & =\left\{\widetilde{S} \in L(\widetilde{U}) \mid v\left(\widetilde{S}^{\widetilde{U}} \cap \widetilde{T}^{\widetilde{U}}\right)=v\left(\widetilde{T}^{\widetilde{U}}\right), \forall \widetilde{T} \in L(\widetilde{U})\right\} .
\end{aligned}
$$

According to the above discussion, the existence of the Shapley values on restricted coalition is possible. It is not hard to prove the following theorem and corollary.

Theorem 32. Let $v \in G^{C a v}(N)$ be a fuzzy capacity game, and let $\widetilde{S} \in L(N)$ be a random fuzzy coalition with nonnegative variable $s=\left(s_{1}, s_{2}, \ldots, s_{n}\right)$; then the Shapley value on restrict fuzzy coalitions $\widetilde{U}$ with nonnegative variable $u=\left(u_{1}, u_{2}, \ldots\right.$, $u_{n}$ ) is as follows:

$$
\begin{aligned}
& f_{i}(v)\left(\widetilde{S^{U}}\right) \\
&=\sum_{i \in T \subseteq S \in P(N)} \frac{(|T|-1) !(|S|-|T|) !}{|S| !} \\
& \quad \times\left\{w_{\widetilde{S^{U}}}(T)-w_{\widetilde{S^{U}}}(T \backslash i)\right\} .
\end{aligned}
$$

Corollary 33. Let $v \in G^{\text {Cav }}(N)$ be a fuzzy capacity game, and $\widetilde{S} \in L(N)$ be a random fuzzy coalition with nonnegative variable $s=\left(s_{1}, s_{2}, \ldots, s_{n}\right)$; then the Shapley value for any $i \in$ $N$ on restricted fuzzy coalitions $\widetilde{U}$ with nonnegative variable $u=\left(u_{1}, u_{2}, \ldots, u_{n}\right)$ can be calculated by

$$
\begin{array}{cl}
f_{i}(v) & \left(\widetilde{S}^{\widetilde{U}}\right)=\max \sum_{T_{j} \subseteq N} \alpha_{T_{j}} f_{i}^{\prime}(v)\left(T_{j}\right) \\
\text { s.t. } \quad & \sum_{T_{j}} \alpha_{T_{j}} 1_{T_{j}}=s^{u}, \\
& T_{j} \subseteq N, \quad\left(j=1,2, \ldots, 2^{n}\right), \\
& \alpha_{T_{j}} \geq 0, \quad\left(j=1,2, \ldots, 2^{n}\right),
\end{array}
$$

where $f^{\prime}$ is the function given in Theorem 4.

\section{Conclusion}

We have proposed an extension of fuzzy cooperative games with fuzzy coalitions by the concave integral so as to address the optimal profits from all subdecompositions of fuzzy coalitions. The proposed class of games is more realistic since it has continuity except for the other properties such as superadditivity and convexity. Meanwhile, the proposed fuzzy game is also an extension of the game with the Choquet integral form defined by Tsurumi et al.

In fuzzy game, the Shapley values of game with fuzzy coalitions are also an important solution concept. Inspired by Tsurumi et al., the general Shapley values for games with fuzzy coalitions are proposed, and the correlation with the crisp Shapley values is discussed. Further, we give the simplified expression by the crisp Shapley values. In fact, as long as the fuzzy coalition variables are given, the Shapley value of a player with a certain participation level can be completely obtained similarly to that of crisp cooperative game.

\section{Conflict of Interests}

The authors declare that they do not have commercial or associative interests that represent a conflict of interests in connection with this paper. Besides, there are no professional or other personal interests that can inappropriately influence the submitted work. 


\section{Acknowledgments}

This research was supported by the National Natural Science Foundation, China (Grant no. 71271029) and Education of Humanities and Social Science Research on Self-fund Project, China (Grant no. 11YJE630003). The authors would like to express their deep gratitude to the referee for his/her valuable comments and suggestions.

\section{References}

[1] L. S. Shapley, "A value for n-person games," Annals of Mathematics Studies, vol. 28, pp. 307-317, 1953.

[2] J. Banzhaf, "Weighted voting does not work: a mathematical analysis," Rutgers Law Review, vol. 19, pp. 317-343, 1965.

[3] S. Tijs, "Bounds for the core and the $\tau$-value," in Game Theory and Mathematical Economics, pp. 123-132, North-Holland Publishing, Amsterdam, The Netherlands, 1981.

[4] L. A. Zadeh, "Fuzzy sets," Information and Control, vol. 8, no. 3, pp. 338-353, 1965.

[5] M. Mareš, "Weak arithmetics of fuzzy numbers," Fuzzy Sets and Systems, vol. 91, no. 2, pp. 143-154, 1997.

[6] D. Dubois and H. Prade, Fundamentals of Fuzzy Sets, The Handbooks of Fuzzy Stes Series, Kluver Academic Publishers, Boston, Mass, USA, 2000.

[7] J. P. Aubin, "Coeur et valeur des jeux flous à paiements latéraux," Comptes Rendus Hebdomadaires des Séances de l'Académie des Sciences A, vol. 279, pp. 891-894, 1974.

[8] D. Butnariu, "Fuzzy games: a description of the concept," Fuzzy Sets and Systems, vol. 1, no. 3, pp. 181-192, 1978.

[9] M. Mareš, "Fuzzy coalition structures," Fuzzy Sets and Systems, vol. 114, no. 1, pp. 23-33, 2000.

[10] M. Mareš, Fuzzy Cooperative Games: Cooperation with Vague Expectations, Physica, New York, NY, USA, 2001.

[11] M. Mareš and M. Vlach, "Linear coalitional games and their fuzzy extensions," International Journal of Uncertainty, Fuzziness and Knowlege-Based Systems, vol. 9, no. 3, pp. 341-354, 2001.

[12] M. Tsurumi, T. Tanino, and M. Inuiguchi, "Shapley function on a class of cooperative fuzzy games," European Journal of Operational Research, vol. 129, no. 3, pp. 596-618, 2001.

[13] S. Borkotokey, "Cooperative games with fuzzy coalitions and fuzzy characteristic functions," Fuzzy Sets and Systems, vol. 159, no. 2, pp. 138-151, 2008.

[14] D. Butnariu, "Stability and Shapley value for an n-persons fuzzy game," Fuzzy Sets and Systems, vol. 4, no. 1, pp. 63-72, 1980.

[15] D. Butnariu and T. Kroupa, "Shapley mappings and the cumulative value for n-person games with fuzzy coalitions," European Journal of Operational Research, vol. 186, no. 1, pp. 288-299, 2008.

[16] R. J. Weber, "Games in coalitional form," in Handbook of Game Theory, R. J. Aumann and S. Hart, Eds., vol. 2, Elsevier, Amsterdam, The Netherlands, 1994.

[17] Y. Azrieli and E. Lehrer, "Extendable cooperative games," Journal of Public Economic Theory, vol. 9, no. 6, pp. 1069-1078, 2007.

[18] J. Dow and S. Werlang, "Uncertainty aversion, risk aversion, and the optimal choice of portfolio," Econometrica, vol. 60, pp. 197204, 1992.

[19] J. Dow and S. R. D. C. Werlang, "Nash equilibrium under Knightian uncertainty," Journal of Economic Theory, vol. 64, no. 2, pp. 305-324, 1994.

[20] E. Lehrer, "A new integral for capacities," Economic Theory, vol. 39, no. 1, pp. 157-176, 2009. 


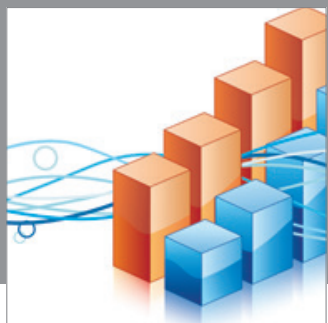

Advances in

Operations Research

mansans

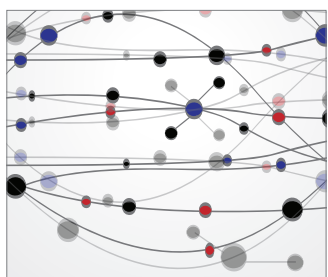

The Scientific World Journal
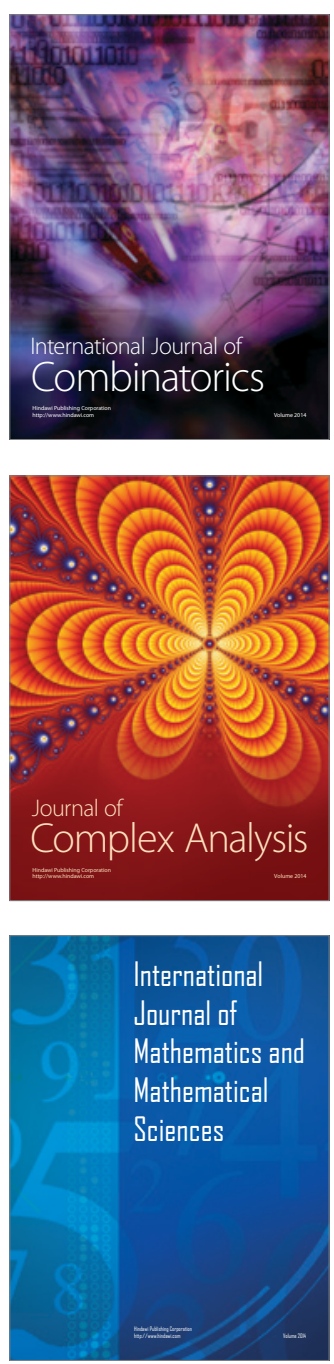
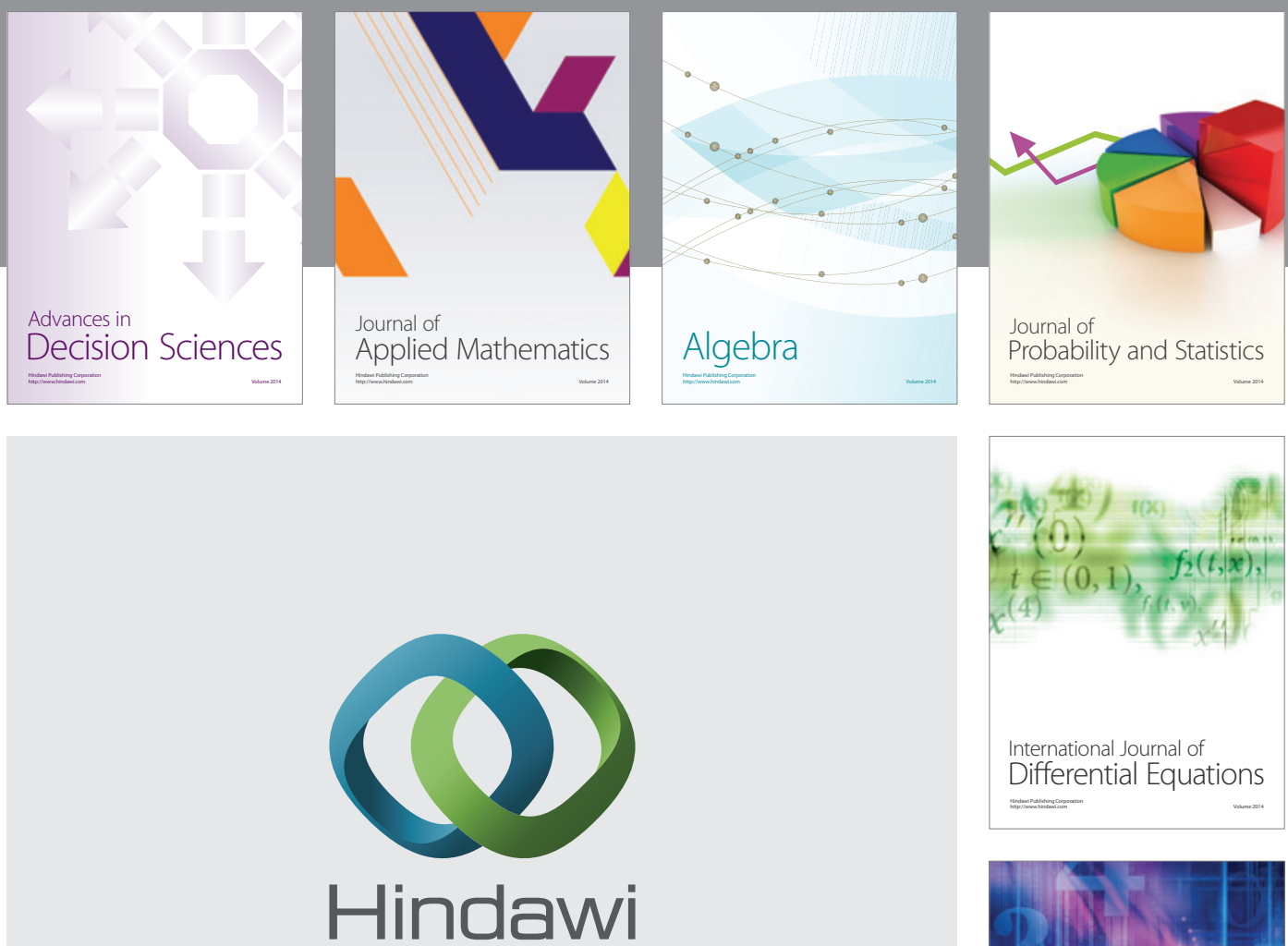

Submit your manuscripts at http://www.hindawi.com
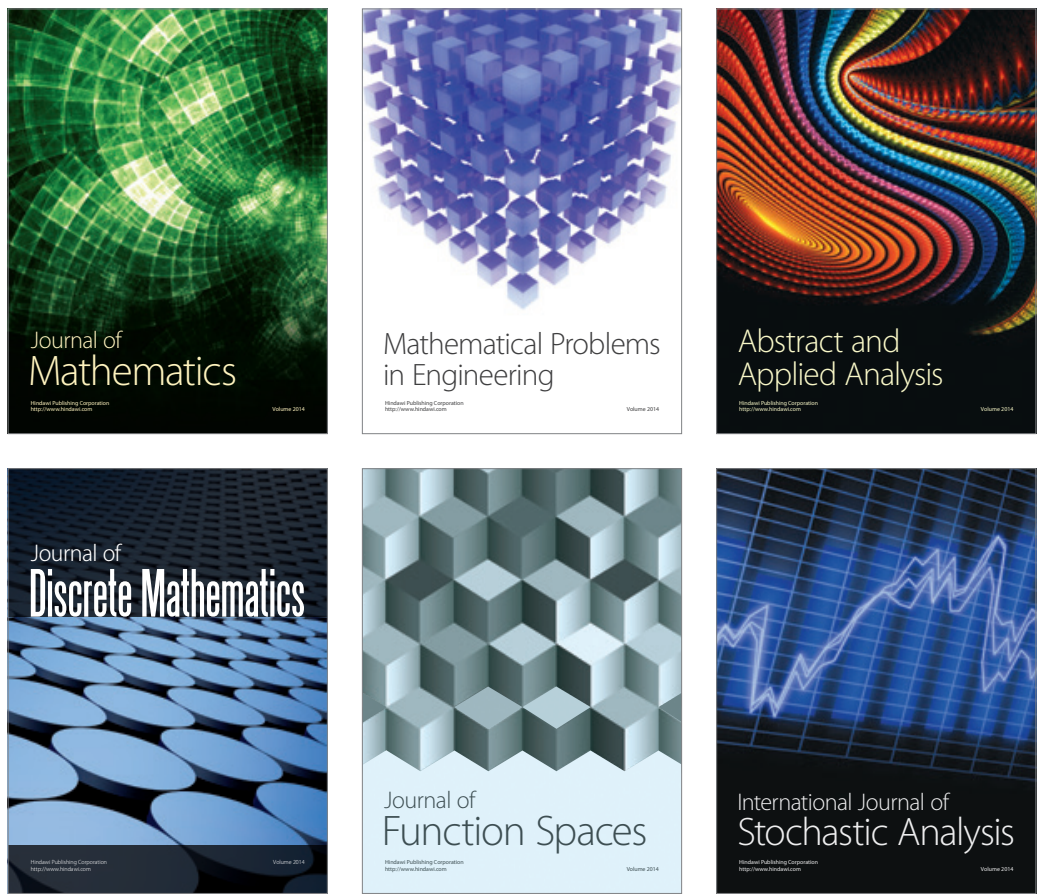

Journal of

Function Spaces

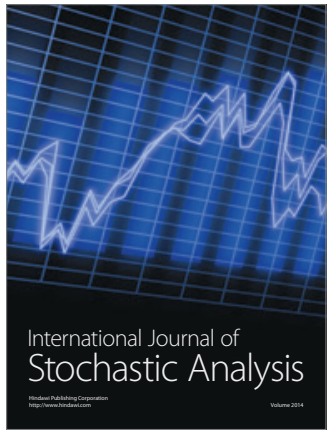

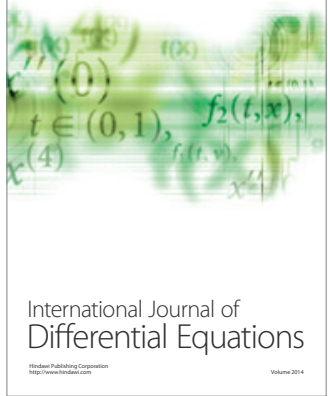
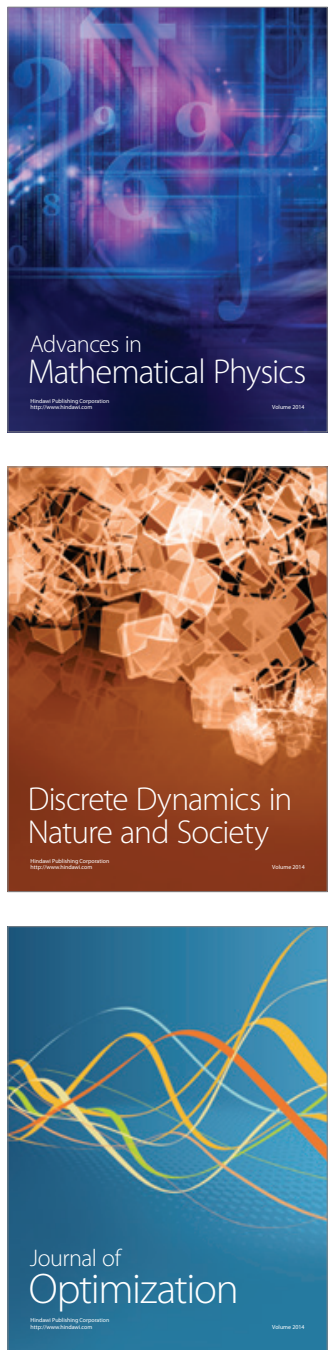